\title{
Röntgenpionier Dr. med. Reinhold Alexander von Sengbusch (1869-1944) \\ (Erinnerungen meines Großvaters)
}

\author{
Werner von Sengbusch
}

\section{Vorwort}

"Sie könnten die Erinnerungen an Ihren Großvater, der hier in Riga segensreiche, medizinische Arbeit geleistet hat doch einmal aufschreiben", so wurde ich gebeten, als ich nach Spuren unserer Familie und insbesondere unseres Großvaters in Rìga und Umgebung suchte und dabei das Pauls Stradiņš Museum für Geschichte der Medizin und das 1. Städtische Krankenhaus besuchte.

Erinnerungen an den Großvater, den ich nur aus Gesprächen und von Bildern her kannte zu schreiben, dazu fühlte ich mich nicht in der Lage. Aber es gab Gott sei Dank einmal einen Archivar des ärztlichen Vereins

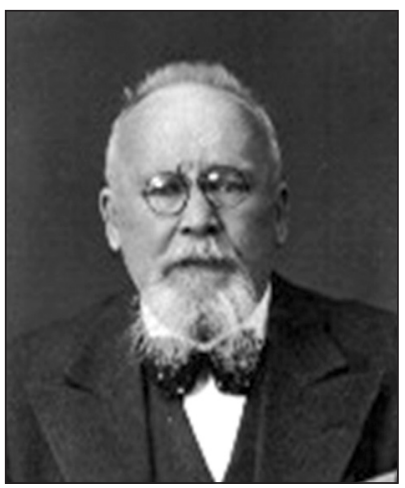
in Rìga, Dr. von Petersenn, der meinen Großvater rechtzeitig gebeten hatte, seine Lebenserinnerungen nieder zu schreiben.

So schrieb er in den Jahren 1931/32 auf, was ihm wichtig war. Leider ist uns nur der erste Teil seiner Lebenserinnerungen erhalten geblieben.

In dieser Veröffentlichung beschränke ich mich auf die Stationen seines Studiums in Dorpat, seinem Aufenthalt in St. Petersburg, Berlin und den Aufbau seines Röntgenkabinetts, Ritterstraße 8b in Rīga.

Sein Medico-mechanisches Institut nach dem Stockholmer "Zander-System" und seine Entwicklung von Prothesen nach dem 1. Weltkrieg sind weitere tech- 
nische Entwicklungen in der Medizin des letzten Viertels des 19. und der ersten Hälfte des 20. Jahrhunderts.

\section{Reinhold Alexander von Sengbusch}

\section{Ein kurzer Familienabriss}

Eigentlich hatte ich nur die Absicht meine Erinnerungen an die Zeit des Weltkrieges niederzuschreiben, dieselben der Familientruhe einzuverleiben, vielleicht, dass in Zukunft jemand sie wieder gelesen hätte. Nun mahnt aber schon wiederholt unser rühriger Archivar des ärztlichen Vereins, Dr. von Petersenn - vor mir liegt sein letztes Schreiben vom Februar 1931 - ihm Lebensbeschreibungen einzusenden.

Geboren bin ich am 26. Juli 1869 Transdüna, im damaligen Thilo'schen Höfchen an der Dünamünd'schen Straße [1] (später stand auf dem Grund die in der Kriegszeit zu Grunde gegangene Fabrik der "Westfälische Drahtindustrie") [2], wo meine Eltern den Sommer verlebten. Unsere Stadtwohnung war damals am Domplatz. Erst 1870 bezogen meine Eltern die 2. Etage der kleinen Sünderstraße Nr. 1 [3] [Mazā Grēcinieku iela]; mein Großvater Wilhelm von Sengbusch [4] bewohnte die untere Etage.

Mein Vater war der Kaufmann erster Gilde, damals auch Schwedischer Vicekonsul Oscar von Sengbusch [5], er war Inhaber der seit 1762 bestehenden Firma A. G. Sengbusch \& Co., sein Compagnon war sein Bruder Carl Gustav von Sengbusch [6], der sich große Verdienste um unser "Altertumsforschendes Museum" [7] erworben hat.

Meine Mutter war Anna Elisabeth geb. Pychlau [8], Tochter des Ratsherrn Reinhold Pychlau [9], der mein Taufvater war.

Nach dem Tode meines Großvaters Reinhold Pychlau übernahm mein Vater die vom Vater des Großvaters gegründete Holzsägemühle Th. Pychlau [10]. Dort lebt jetzt seit 1921 mein jüngerer Bruder Alexander [11]. Die Firma und die Fabrik wurden im Kriege [12] liquidiert, der große, schöne Fabrikschornstein fiel erst vor einigen Jahren. Von 1915-18 war dort die Sommerresidenz meines verstorbenen ältesten Bruders Oscar [13].

\section{Studentenzeit in Dorpat}

Im Januar 1890 ging ich nach Dorpat, mit vielen Ermahnungen von Seiten der Eltern, namentlich von Mutter, die, glaube ich, vielleicht nicht zu Unrecht, etwas besorgt war, ob ich nicht zu viel Dummheiten dort machen würde, hatte ich ja auch schon als Gymnasiast mir Einiges geleistet.

Das corporelle Leben habe ich in den ersten Semestern ausgiebig genossen. Wenn ich an mein Studium denke, vielleicht etwas zu sehr, freilich habe ich die Examina doch noch stets zur richtigen Zeit und auch nicht schlecht abgemacht, 
habe bei ihnen aber auch sehr viel Glück gehabt. Die erste Hälfte des Philosophykums machte ich mit "sehr gut".

Erst im dritten Semester habe ich regelmäßiger die Collegien besucht. Nach Schluß des vierten Semesters habe ich dann mit Oscar Schabert in der Soborowskerei gelebt und mich nur mit meinem Studium beschäftigt, Schabert stand vor dem Schlussexamen, das er auch damals bestand. Wir haben wirklich fabelhaft gearbeitet, alle zwei Stunden in einer kleinen Pause wurde auf dem Hofe zehn Minuten Kurnik oder Katschki gespielt, auch ein Spiel das heutzutage gar nicht mehr gespielt wird. Ganz selten wurde aber auch damals etwas gebummelt, einige Male tüchtig gefeiert, was dem Endresultat eines guten Examens zum Glück nichts geschadet hat.

1893 war ich Conventskassenvorsteher, ein Amt, das nur am Anfang und am Ende des Semesters bei den Zahlungsterminen einem Zeit nahm. Von diesem Semester an habe ich ernstlich angefangen zu studieren, waren es doch auch die viel interessanteren klinischen Semester, die jetzt begannen; auch die Zeit des Arbeitens im Anatomikum, eine Beschäftigung, die mir nur in den ersten Tagen nicht ganz angenehm war.

Gelebt habe ich fast alle die Studentenjahre über mit Carl Brutzer [14] und Gerhard Kieseritzki zusammen, auch Cristel Schwartz-Pölwe war ein Jahr mein Stubenflausch in der Botanischenstraße 4, der Soborowskerei; auch er war damals schon verlobt, was zum Ernste unserer Arbeit viel beitrug.

Ich hatte mich 1893 in Riga mit Hanna Becker [15] (der Vater war Direktor der Riga-Dünaburger Eisenbahn) verlobt. Ich kannte und interessierte mich schon als Schüler stark für sie, wir waren, als ich Primaner war, fast unzertrennliche Läufer auf der Schlittschuhbahn. Auch verkehrte ich schon früher oft in Oger [Ogre] bei Beckers. Diese Verlobung war hauptsächlich der Grund, weshalb ich es plötzlich sehr ernst mit meinem Studium nahm; freilich kam die Tatsache hinzu, dass die Russifizierung immer mehr Fortschritte machte, dass mehrere Examina schon in russischer Sprache gemacht werden mussten, welches ich damals nur sehr mangelhaft beherrschte. Man musste sich also beeilen, um noch die Fächer, die in Deutsch zu absolvieren möglich waren, abzumachen.

Einige Fächer habe ich bereits in Russisch gemacht, so die innere Medizin. Ein Semester vorher hatte Professor Unverricht Dorpat verlassen, sein Nachfolger Wassiljew war ein ganz gemeiner Kerl. Es hatten sich in der Zeit alle anständigen Professoren Russlands geweigert den Ruf nach Dorpat anzunehmen, da sie der Meinung waren, dass es eine Schmach sei, den guten Ruf der Universität durch dieses Manöver zu Grunde zu richten. Nur wirklich Minderwertige ließen sich bereit finden für hohes Geld hier Stellungen zu übernehmen. Diesen Herren waren aber gerade die corporellen Studenten ein besonderer Dorn im Auge, so dass man schon besonderes Glück haben musste, ohne mehrfache Wiederholung, die Fächer zu bestehen. 


\section{W. von Sengbusch}

Auch Gubaroff, der Nachfolger von dem berühmten Prof. Küstner, dem Geburtshelfer und Gynäkologen, der nach Breslau berufen wurde, war so ein Sch.... Etwas anständiger in seiner Gesinnung war Affanasieff, der Nachfolger vom berühmten und sehr interessanten Thoma, bei dem ich das Colleg gehört hatte, aber nicht mehr das Examen machen konnte. Brutzer und Kieseritzki, die sich fleißiger mit dem Fache schon früher beschäftigt hatten, machten dieses Fach noch bei Thoma ab, und wurden von mir sehr beneidet, da sie dadurch einen bedeutenden Vorsprung vor mir erzielt hatten, den ich freilich später gut einholte.

Das letzte, das schwere Examensjahr lebte ich wieder mit meinen alten Cöten Brutzer und Kieseritzki im Wallgraben 7, der Engelharderei, einer Burg, die immer von Medizinern, die zum Schlussexamen arbeiteten, bewohnt wurde. Es war eine sehr hübsche Burg, hatte drei gute Zimmer, eine gute Aufwärterin, die sogar recht sauber war, auf die Wäsche gut aufpasste, nicht stahl, was aber bei den wirklich meist vorzüglichen, estnischen Aufwärterinnen auch sonst kaum vorkam, sie kochte auch gutes Abendessen, so dass wir uns sehr gut einlebten. Mittags wurde nie in den Studentenburgen gegessen, es waren besondere Speisehäuser dazu da, wo sich eine Anzahl L. L. zusammenfand, die die gleiche Mittagsstunde einhalten konnte.

Mein Examen bei Affanasieff ging wohl sehr schlecht, zum großen Teil wohl wegen meiner Unkenntnisse in der russischen Sprache, aber freilich wohl auch wegen der Unkenntnisse im Fache selbst. Der Professor ließ mich allerdings durch, aber mit dem ihm durch Handschlag gegebenen Versprechen, mich nicht später als Anatomischer Pathologe zu beschäftigen, was ich ihm damals mit ruhigem Gewissen versprach, eigentlich aber ein sehr merkwürdiges Verlangen von ihm, das ich ihm aber gerne erfüllte.

Bei Prof. Rauber hatte ich mich im Präparieren von Leichenpräparaten sehr bemüht und sehr fleißig gearbeitet, sowohl bei den Muskel-, als auch in dem späteren Semester an Nervenpräparaten; beim Schluss des Semesters hatte ich zwei Präparate mehr gemacht, als eigentlich nötig war, war aber mit dem letzten nicht ganz fertig geworden. Rauber wollte mir dasselbe bis zum Anfang des nächsten Semesters aufbewahren lassen, damit ich es beenden könne.

In Rìga herrschte nun damals die Cholera, ich war aufgefordert worden, als Unterassistenzarzt an Stelle von Dr. Weidenbaum einzuspringen. Dr. Bertels war Leiter der Abteilung am Stadtkrankenhause, Dr. Max Ulmann Assistenzarzt. Ich nahm gerne an, wollte aber freilich zu Semesteranfang wieder nach Dorpat, meine Abfahrt verzögerte sich aber durch die Erkrankung von Bertels, so kam ich erst Ende September nach Dorpat, meldete mich auch bald bei Rauber. Er war sehr ungehalten, konnte es scheinbar gar nicht verstehen, dass ich die Arbeit bei Kranken der Beendigung meines Präparates vorgezogen hatte.

Ich hätte aber auch gar nicht mehr im Anatomikum arbeiten können, da ich mir fest vorgenommen hatte, im Mai 1895 zum Schlussexamen vorzugehen, aber noch die Chirurgische Klinik und anderes Wichtiges abzumachen hatte. Die Her- 
ren Assistenzärzte im Rigaer Städtischen Krankenhause, wie: Paul Klemm, Buchholz, Manni Kröger und andere, mit denen ich viel zusammengekommen war während meiner Arbeitszeit in der Cholerabaracke, schüttelten alle die Köpfe, sie erklärten es für unmöglich, dass ich schon vorgehen wolle. Eigentlich nur Ulmann hat mir zugeredet es zu versuchen, ich studierte dann ja erst freilich 5 1/2 Jahre, dieses war der allerfrüheste Termin für einen Mediziner. Unheimlich und unwahrscheinlich erschien mir die Sache freilich auch, aber auf den Versuch wollte ich es ankommen lassen.

Der kühne Versuch gelang auch wider Erwarten und zwar recht gut. Prof. Rauber hat mir die obige Sache zwar doch übel genommen, er ließ mich beim ersten Male nicht durch, gab mir aber einen zweiten Termin nach ca. 14 Tagen, wobei dann die Sache klappte. Als ich dann nach überstandenem letzten Examen in die Kanzlei ging, um meine Urteile zu erfahren, erfuhr ich, dass Rauber schon nach meinem ersten Versuche des Examens mir sein Urteil mit "genügend" eingetragen hatte. Ich hätte mich also gar nicht so anzustrengen und aufzuregen gebraucht.

Nach Beendigung meines Studiums hatte ich fest darauf gerechnet am Rigaer Städtischen Krankenhause als Assistenzarzt anzukommen, es war aber leider keine Vakanz vorhanden, auch absolut keine Aussicht auf baldiges Freiwerden einer Stelle. Es war noch eine ganze Reihe von jungen Medizinern, die sich vor mir gemeldet hatten. Ich rechnete freilich damit, dass ich ein Vorrecht haben würde, falls eine Stelle frei wird, da ich doch recht lange Zeit schon vorher in der Cholerabaracke für Gotteslohn, d. h. ohne Gage gearbeitet hatte.

Nachdem ich ca. 1 1 $\frac{2}{2}$ Monate mich zu Hause von der Anstrengung Dorpats erholt hatte, machte ich nun im Krankenhaus die Visite bei Dr. Hampeln mit, bei dem man auch so viel lernen konnte, auch die Sektionen, die damals Dr. Kranhals machte, sah ich mir regelmäßig an.

\section{Aufenthalt in Petersburg und Berlin}

College Albanus, der mit mir zusammen fertig geworden war, war nach Petersburg gegangen und hatte am Obuchow-Hospital, und zwar an der weiblichen Abteilung, die der berühmte Internist Dr. Kernig leitete, eine Assistenzstelle bekommen. Er hatte durch seinen verstorbenen Vater, der auch Hofarzt gewesen war, gute Beziehungen dahin gehabt. Ihn nun hatte ich gebeten, mir Nachricht zu geben, ob Aussicht sei, dass ich eventuell dort eine Anstellung, wenn auch nur als Volontärassistent bekommen könne. Nun schrieb er mir, er hätte mit Kernig meinetwegen gesprochen, ich solle so bald wie möglich herüberkommen, durch Dr. Kernig würde es mir sicher gelingen im selben Hospitale, allerdings ungagiert anzukommen; er teilte mir noch mit, dass er schon eine Menge gesehen und zugelernt hätte.

Da es mir auf ein Gehalt damals wenig ankam, wohl aber eine Stelle zu finden, wo ich möglichst viel und verschiedenes Krankenmaterial sehen könnte, griff ich 


\section{W. von Sengbusch}

sofort zu, habe es auch nicht bereut, obgleich ich wohl glaube, dass ich als ordentlicher Assistent hier in Rīga noch mehr gelernt hätte.

Bald hatte ich im Hospital in Petersburg aber schon eine kleine Abteilung von acht Betten, die ich allein zu versorgen hatte. Ich machte täglich die Visite mit, die Dr. Kallmeyer, mein direkter Vorgesetzter in den inneren Baracken machte. Dr. Kernig der Chefarzt über das Krankenhaus war, kam nicht täglich in die Abteilung, die Kallmeyer unter sich hatte, da letzterer ein alter, erfahrener Kollege war, er hatte es aber gerne, wenn man ihn aufsuchte und sich seinem Rundgang anschloss, er hatte aber auch leider sehr viel in der Kanzlei zu tun. Am Krankenbette habe ich sowohl seine Art und Weise, mit den Kranken zu verkehren, als auch sein fabelhaftes Wissen und seine Diagnosestellung bewundern gelernt. Er war dabei bescheidenen Charakter und von großer Liebenswürdigkeit.

1896 schließt sich ein Aufenthalt in Deutschland, in Berlin an. Da ich damals noch den Plan hatte, Landarzt zu werden, hörte ich dementsprechend die verschiedensten Collegien, auch nahm ich praktische Kurse, z. B. gynäkologische und auch einen geburtshilflichen, "Leyden", "Senator", "Dührssen", den Kinderarzt "Heubner", später auch "Ernst von Bergmann" habe ich gehört. Von 8 Uhr früh bis zum Mittag habe ich die Vorlesungen besucht, nach dem Mittag wurde dann zu Hause das Gehörte nachgearbeitet. Am Abend war man aber zu anderen Dingen frei.

Es waren in der Zeit recht viele Balten auch gerade zum Studium in Berlin, so: Otto Stender, Maler Krauss, Manni Kröger, Buchholz, Scheuber. Wir haben sie öfter getroffen und sie auch bei uns zum Abend gesehen.

Am 19. März 1897 alten Styles kamen wir wieder in Riga an, zogen, da wir ja noch keine Wohnung hatten, in die kleine Sünderstraße 1, drei Treppen hoch, in das frühere Zimmer von Tante Saschinka Boetticher. Wir haben dann bald in der Dorpaterstraße 13 [Térbatas iela], eine sehr gute Wohnung gefunden.

Verschiedentlich Unterhandlungen wegen einer Anstellung aufs Land hin, habe ich gehabt, es fand sich aber nichts Geeignetes. Eine Stelle nach Adiamünde war die letzte, die mir 1898 angeboten wurde, doch wurde mir damals schon von vielen Collegen stark abgeraten aus Rìga fort zugehen. Ich gab damals diesen Plan dann definitiv auf.

\section{Niederlassung in Rīga}

1898 bekam ich von der damals eben gegründeten "Gegenseitigen Unfallgesellschaft", aus der sich die jetzt noch bestehende "Allgemeine Unfallgesellschaft" entwickelt hat, die ersten Patienten zur Massagebehandlung zugeschickt, selten einmal verirrte sich auch ein Privatpatient zu mir.

Bald nachdem ich mich niedergelassen hatte, fing ich an, in der Kinderambulanz zu arbeiten. Dieselbe war zur Erinnerung an Dr. August Haken in der Schulenstraße [Skolas iela] gegründet worden. Die Chirurgische Abteilung leitete 
Dr. Paul Klemm, Ado Helmboldt [16] arbeitete unter ihm, nachdem er aus Lemburg, wo er Landarzt gewesen war, nach Rīga übergesiedelt war.

Die Innere Abteilung hatte Dr. C. Deubner unter sich. Es arbeiteten bei ihm F. Buchholz [17] und Edgar May. Die Ohren- und Nasenabteilung gründete daselbst Mühlen.

Erst hospitierte ich bei Deubner in der Inneren Abteilung, bald aber ging ich in die Chirurgische über, machte die Narkosen, half bei den Verbänden, machte die nötigen Massagen, elektrisierte und bemühte mich, mich auch sonst nützlich zu machen. Aus der Kinderambulanz entwickelte sich bald im Interesse der "Gegenseitigen Unfallgesellschaft" die Ambulanz für Unfallverletzte. Ambulant wurden freilich auch Kinder weiter empfangen; die einer stationären bedürftigen Kinder, aber ins neu eröffnete Armitstead'sche Kinderhospital [18] geschickt. Dort übernahm auch Klemm die Chirurgische Abteilung.

In der Schulenstraße kam es zum Bau eines modernen Operationszimmers im Hinterhause und wurde vorne noch eine Etage aufgestockt, so dass Platz, glaube ich, für 18-20 Betten geschafft wurde. Es war ein angenehmes, kollegiales $\mathrm{Zu}$ sammenarbeiten, ich habe viel dort gelernt. In der kleinen Chirurgie konnte ich späterhin selbständig arbeiten, diese Zeit ist mir wesentlich im Kriege nützlich gewesen. Meine Spezialität wurden mit der Zeit die Schulterluxationen, die recht häufig vorkamen.

Ich versuchte es, die Nachbehandlung möglichst herabzusetzen, indem ich die Ruhigstellung des Oberarmes stark einschränkte, meist fing ich schon am zweiten Tage mit Massage und leichten Bewegungen an. Die Erfolge waren so sehr gute, stachen wesentlich von den Krankenhauserfolgen ab, wo die Fälle meist für wenigstens zwei Wochen in einen festen Verband gelegt wurden, was leider auch jetzt vielfach geschieht und dadurch eine oft Monate lang dauernde, energische Nachbehandlung erforderlich wird, die aber oft auch nicht ganz zum Ziele führt. Ebenfalls der Fracturbehandlung nahm ich mich mit Liebe an, auch da führte ich frühzeitige Massage mit sehr gutem Erfolge ein.

Nachdem ich mir mein Röntgeninstitut als erster Privatarzt in Rīga eingerichtet hatte, kam als meine weitere Spezialität die Entfernung der oft vorkommenden Fremdkörper hinzu. Ich erinnere mich manchmal an einem Tag 2-3 Nadelspitzen aus Fingern oder der Hand entfernt zu haben. Ado assistierte mir dabei und hatten wir uns eine große Gewandtheit dabei angelegt. Es war eine Arbeit, die merkwürdigerweise Klemm gar nicht lag, ich habe öfter von ihm angefangene Fälle, wo er den Fremdkörper nicht finden konnte, zu Ende operiert; auch hatte ich mir ein so feines Tastgefühl angelegt, dass ich Fremdkörper in der Tiefe abtasten konnte, die andere absolut nicht fühlten. Einige Male habe ich daher auch ohne Röntgenbild sofort die Incision mit Erfolg gemacht.

Im Jahre vor dem Weltkrieg zogen wir in das neu gebaute, mit allem Komfort ausgestattete, groß angelegte Krankenhaus [19] an der Paulskirche. Das Einzige, 


\section{W. von Sengbusch}

was fehlte, war ein Röntgenkabinett, was mir aber nicht leid tat, da so alle Fälle zum Röntgen auch weiterhin zu mir geschickt wurden.

Auch nach Eröffnung des Kinderhospitals habe ich noch durch Jahre alle Röntgenaufnahmen für dort gemacht. Im Laufe der Jahre habe ich zufällig bei Verbänden in der Ambulanz vier Fälle von Lepra nerv. diagnostiziert, die alle dann erst in der Inneren Abteilung und nachher noch von Dr. von Bergmann dem großen Spezialisten für Lepra, als solche bestätigt worden sind. Mein Blick für diese Fälle wurde namentlich von Schwager Buchholz rühmlichst anerkannt, umso mehr als in derselben Zeit in der Inneren Abteilung nur ein einziger Fall entdeckt worden war, doch spielt ja der Zufall bei solchen Dingen viel mit.

\section{Aufbau einer eigenen Röntgenpraxis}

Nun will ich berichten, wie ich dazu kam, mich für die Röntgensache zu interessieren. Der Physiker Pohrt hatte bald nach Entdeckung Röntgens auch in Rīga angefangen zu experimentieren, dann schaffte sich 1898 die Stadt fürs Krankenhaus einen Apparat an, den Pohrt bediente. Es kam aber leider sehr wenig Erfreuliches dabei heraus, er war ja auch gar nicht in Deutschland oder irgendwo draußen zum Lernen gewesen, hatte nur selbst seine Studien getrieben. Wenn auch leichte Aufnahmen gelangen, versagte die Technik vollkommen bei etwas schwierigeren Fällen.

\section{Erste Erfahrungen}

Vetter Herbert Schmidt [20] aus Pernau hatte in der Zeit in Russland in einem Kürassierregiment gedient, er war bei einem Kaisermanöver, als Ordonnanzreiter in Gegenwart seiner Majestät und seines ganzen Stabes schwer verunglückt, indem er mit einer anderen reitenden Ordonnanz in voller Karriere zusammenprallte und angeblich für tot erst nach vielen Stunden in ein Hospital transportiert worden ist. Er lag dort viele Wochen lang bewusstlos an den Folgen eines Schädelbruches, offenbar einer Basisfractur.

Tante Lina [21] war aus Pernau gleich nach der Benachrichtigung über den tödlichen Unfall nach Moskau gefahren, hatte alles in Bewegung gesetzt, ihn in eine Privatklinik überführen zu dürfen. Es wurde mit der Zeit konstatiert, dass nebenbei auch eine schwere Verletzung des Schultergelenkes vorgelegen hat, die bis dahin gar nicht bemerkt worden war. Als er nach vielen Wochen, resp., Monaten transportfähig war, brachte sie ihn nach Rigga. Er kam in die Ambulanz zur Untersuchung, es bestand eine totale Versteifung des Schultergelenkes mit hochgradigen Schmerzen, namentlich auch bei den leichtesten passiven Bewegungsversuchen. Der Oberarm war stark adduciert, die Muskulatur atrophisch, die Gelenkpfanne schien uns leer zu sein, es wurde die Diagnose der Luxation gestellt, die starken Schmerzen auf Nervendruck bezogen.

Damals wurden die ersten Röntgenaufnahmen dieses Falles bei Pohrt versucht; sie scheiterten leider kläglich. Es konnte nur konstatiert werden, dass die 
Pfanne, so wie wir es angenommen hatten, leer war, über einen weiteren Knochenbefund ließ sich aber trotz mehrfacher Wiederholung der Aufnahme nichts erfahren. Es war das Bild einer reinen Mondlandschaft, die Knochen hoben sich gar nicht von der Muskulatur in der Zeichnung ab. Die Stellung des Oberarmes ließ neben der Luxation einen Bruch des Oberarmes vermuten, doch konnte auch nicht mit einiger Sicherheit angenommen werden, wo der Bruch saß und wie er durch den Knochen verlief.

Klemm entschloss sich den Versuch einer Reposition zu machen. In tiefer Narkose wurden vorsichtige Repositionsversuche gemacht, der Erfolg war, dass es einen deutlichen Krach gab, der Arm in Narkose bedeutend mehr zu bewegen war. Handelte es sich nun um Verwachsungen, die wir mit dem Krachen gelöst hatten oder war eine Refractur eingetreten? Das letztere war wahrscheinlicher und bestätigte sich auch später durch den Verlauf des Falles. Es trat unter dem Verbande eine sehr starke Schwellung auf, gegen Abend auch sehr starke Schmerzen.

Ich hatte ihn mit Vaters Erlaubnis, in der kleinen Sünderstraße, oben im letzten Zimmer untergebracht, habe auch die drei ersten Nächte bei ihm gewacht. In der ersten Nacht halfen weder Applikation einer Eisblase, noch schmerzstillende Medikamente, nur sehr langsam ließen die Schmerzen nach. Seine Verzweiflung war groß und trug er sich auch nachher noch mit Selbstmordgedanken. Er hatte sich vorgenommen, Ingenieur zu werden, war verzweifelt bei dem Gedanken, dass es ihm nicht möglich sein würde mit seiner verletzten, freilich rechten Hand etwas zu leisten.

Durch regelmäßig viele Wochen lang fortgesetzte Massage hatte er doch einige, auch aktive Beweglichkeit in der Schulter erreicht. Bei der weiteren Behandlung ist es mir auch klar geworden, dass es sich um den abgebrochenen Kopf des Oberarmes handelt, der vorne unter dem Schlüsselbein fühlbar war und die Schmerzen durch Druck auf die großen Nerven machte. Dieser abgebrochene Kopf blieb also sehr störend, ist nach Jahren dann von Prof. Gocht in Deutschland entfernt worden, wobei es zu einer sehr schweren Blutung aus einem großen Gefäß, das mit der Kapsel verwachsen war, gekommen ist. Im Endresultate kann Herbert jetzt den Arm bis etwas zur Horizontalen heben und behindert er ihn fast gar nicht.

Durch das völlige Versagen des Röntgenverfahrens im Krankenhause noch in anderen etwas schwierigeren Fällen, kam ich damals auf die Idee da mich die Sache auch interessierte - mich zum Spezialisten im Fache auszubilden. Ich nahm mir aber gleich vor, nur wenn ich meine Sache sicher beherrschte, damit anzufangen.

Im Krankenhause war ein Apparat von Reiniger, Gebbert \& Schall, ein 50 $\mathrm{cm}$ Inductor installiert, also ein Apparat mit dem man sicher damals sehr Gutes hätte leisten müssen; tatsächlich habe ich später noch einige Jahre gut mit ihm gearbeitet.

Am 14. Mai 1899 fuhr ich für zwei Monate hinaus, habe zuerst in Berlin bei Immelmann, zuletzt auch einige Wochen bei Albers-Schönberg in Hamburg gearbeitet. Ersterer gab schon einige Semester Kurse im Röntgenfache. Er war, als ich 


\section{W. von Sengbusch}

bei ihm anfing, noch sehr bescheiden eingerichtet. Schön war es, dass er sich während meiner Tätigkeit dort ein neues, viel größeres Institut in der Lützowstraße ausbauen ließ und hatte ich so das Glück beim Umzuge dabei zu sein, namentlich aber bei der Montage auch eines ganz neuen, modernen Apparates stets zugegen sein zu können. Dieses ist merkwürdigerweise von keinem der anderen am Kurse Teilnehmenden ausgenutzt worden. Mir brachte es einen wesentlichen Vorteil auch für spätere Zeiten.

$\mathrm{Da}$ ich mit der Montage so absolut vertraut wurde, konnte ich beim Versagen des Apparates leicht selbst die Fehlerquelle finden. In Rìga hatten wir ja auch in den ersten Jahren keinen Spezialisten, der sich mit Montage, resp., Reparatur befasste. Ich habe damals fleißig mitgearbeitet, auch bei Einrichtung der Dunkelkammer, die im Großen Style sehr bequem eingerichtet wurde. So waren nun bei ihm zwei Apparate in Tätigkeit, von denen der Alte mehr zu Durchleuchtungen, der Neue zu Aufnahmen benutzt wurde. Mit Immelmanns Erlaubnis war ich nicht nur die zwei Stunden, die der Kursus dauerte in seinem Institute, sondern blieb dort beinahe den ganzen Tag, oder kam in den Abendstunden wieder. Er war mir ja auch sehr dankbar für alle Hilfe, die ich beim Umzuge ihm geleistet habe. Alle seine vielen Röhren habe ich selbst hinübergetragen, kein einziger Verlust war zu verzeichnen. Er sagte mir zum Abschied, dass er der Überzeugung sei, ich hätte das richtige Verständnis dazu, die nötige Geduld und auch schon, was sehr wichtig sei, die glückliche Gabe geduldig in der Dunkelkammer zu arbeiten, was er leider bei den meisten seiner Schüler vermisse.

In Hamburg hatte Albers-Schönberg im Georgshospital, ein sehr schönes, großes Kabinett und außerdem ein Privates. Er beschäftigte sich schon damals nur mit dem Röntgenfache, während Immelmann nebenbei sich noch einen Gymnastiksaal aufgebaut hatte. In dem privaten Kabinett arbeitete bei Albers auch Prof. Hahn, der Spezialist für Hautkrankheiten war, sich auch auf therapeutischem Gebiete anfing mit der Strahlentherapie zu beschäftigen, ich lernte hier die ersten tastenden, vorsichtigen Versuche kennen.

Auch sah ich hier zuerst den elektrolytischen Unterbrecher, der eine sehr starke Verkürzung der Aufnahmen ermöglichte, die damaligen Röhren freilich über Gebühr angriff. Erst später sind dann Röhren konstruiert, die die großen, durch ihn erzeugten Energien gut vertrugen, eine Änderung der Induktion in der primären Spule war bei den damaligen Apparaten noch nicht möglich, daher die hohen Energien für die Röhren geradezu gefährlich.

Die heutigen Kollegen, die sich auf diesem Gebiete zu beschäftigen anfangen, werden wohl lächeln, wenn sie dieses zu lesen bekommen, daraus ersehen, welche Schwierigkeiten ich damals überwinden musste, bis ich mich in Rigga eingerichtet habe.

Ich entschloss mich damals auch zu einem Induktionsapparat von Reiniger, Gebbert \& Schall, der damals führenden Firma auf diesem Gebiete. Ich habe meine Wahl nicht bereut, nach zwei Jahren habe ich die Primärspule umbauen 
lassen und schaffte mir auch einen 3-stiftigen elektrolytischen Unterbrecher an, den ich dann nach Wahl neben dem Quecksilberunterbrecher benutzen konnte. Der Apparat leistete sehr Gutes, die Collegen in Riga sind mit meinen Arbeiten stets zufrieden gewesen.

Ich habe die Möglichkeit gehabt nach Anschaffung der geeigneten Nebenapparate, Herz- und Lungenaufnahmen in zwei Meter Entfernung, also unvergrößert, zu machen, und zwar bei angehaltenem Atem, in ca. 2-3 Sekunden. Solche Aufnahmen hängen zur Erinnerung an diese schöne Arbeitszeit noch einige im Institute. Für damalige Verhältnisse im Jahre 1907 waren dieses sehr gute Resultate, später mit meinem großen Idealapparate habe ich natürlich noch viel kürzere und auch schärfere Aufnahmen machen können.

1914 nach meiner Einberufung habe ich meinen alten Apparat dem Roten Kreuze in Riga verkauft, habe ihn damals im Krankenhause bei der Paulskirche noch selbst montiert, auch die ersten Aufnahmen dort gemacht, um zu zeigen, dass er richtig und gut arbeitet.

Mein Röntgenkabinett richte ich selber ein.

Damals, als ich mir mein Röntgenkabinett einrichtete, war es noch nicht so einfach wie heute, wo man bei der Bestellung nur anzugeben hat, welchen Stromanschluss man besitzt: Gleich- oder Wechselstrom, dazu die Spannung. In Rīga war noch kein Städtisches Elektrizitätswerk vorhanden, nur wenige Häuser und Fabriken hatten ihre eigene Zentrale, meist Sauggasmotore mit AkkumulatorenAnlagen, die für den Betrieb in der Nacht bestimmt waren. Letzterer wurde geladen, solange der Sauggasmotor tags in Betrieb war.

Nach meiner Rückkehr nach Rigga musste ich lange Verhandlungen führen mit Fachleuten, was das Praktischste für mich sein würde. Ich hätte mich mit einer im Keller eingebauten Akkumulatoren-Anlage behelfen können, diese hätte aber von Zeit zu Zeit aufgeladen und daher transportiert werden müssen. Dies, wäre nicht nur weitläufig, sondern auf die Dauer auch schädlich für die Batterie gewesen. Die nächste elektrische Anlage von mir aus Alexanderstraße [Brìvības iela] 51, war die Brauerei Strytzki in der Nikolaistraße [Valdemāra iela], ein gutes Ende von mir entfernt. Von dort bezog das Krankenhaus den Strom für sein Kabinett. Was sich aber die Stadt erlauben konnte, ich meine die Überführung der elektrischen Starkstromleitung über die Straße, war einer Privatperson lange noch nicht gestattet. Wie mir von Kennern gesagt wurde, solle ich auch gar keinen Versuch machen, die Genehmigung dazu zu bekommen; mit Herrn von Strytzki hatte ich gesprochen, er hatte mir den Strom bereitwillig zur Verfügung gestellt, wie aber ihn zu mir leiten? Ado Helmboldt, der sehr gut mit der ganzen Feuerwehr bekannt war, wies mir den richtigen Weg, er machte mich mit dem Ingenieuren Benjamin bekannt, der hatte damals das ganze Meldewesen unter sich. Neben der telefonischen Meldung hatte die Wehr verschiedentlich telegrafische eigene Leitungen in den Vorstädten, solch, eine Leitung führte durch das Terrain des Krankenhauses, weiter die Schulenstraße entlang. Nun erbot sich Benjamin für 


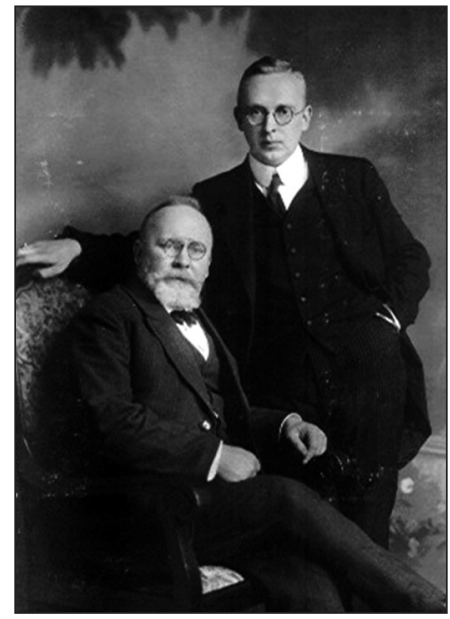

Abb. 1. 1923 - Dr. med. Reinhold Alexander von Sengbusch mit seinem Sohn Reinhold (Reini)

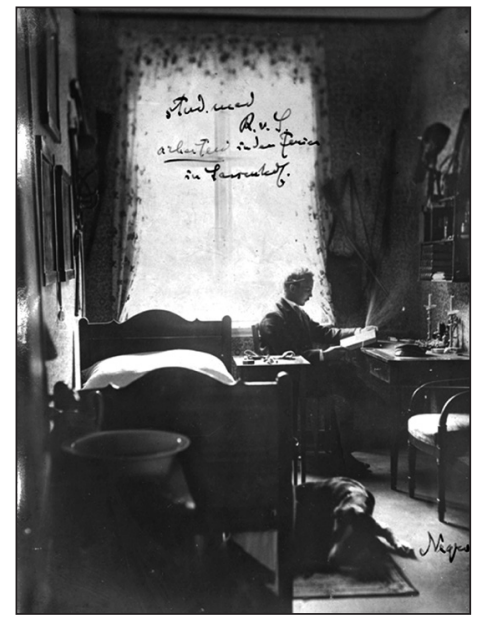

Abb. 2. Studierzimmer während der Ferien in Sassenhof - Gartenstraße 3

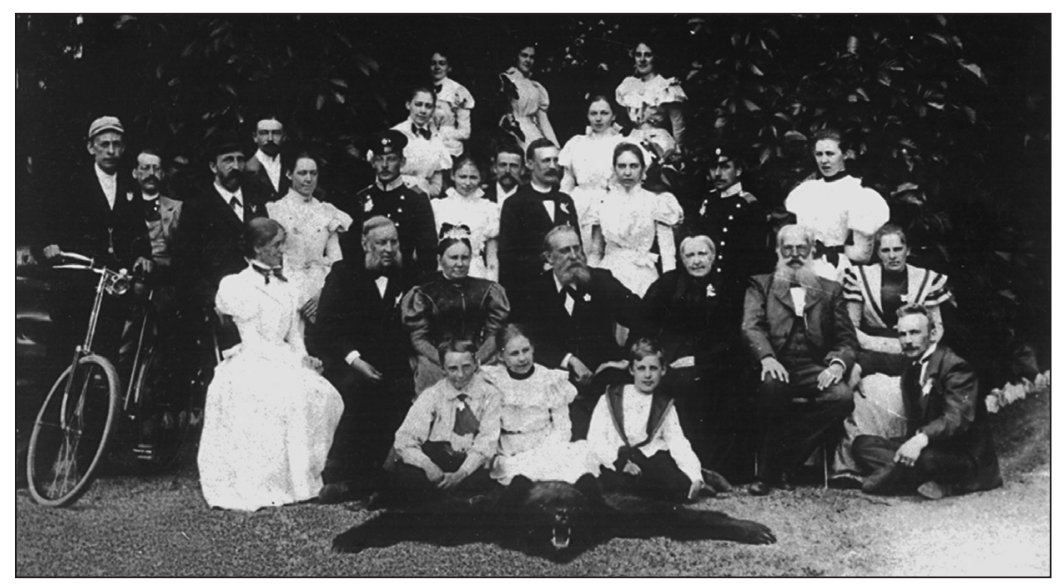

Abb. 3. Silberhochzeit von Onkel Gustav und Genevra v. S. 28.9.1890 in Sassenhof Gartenstraße 3, vor der Veranda, vorne v. l. sitzend Willy v. Boetticher, Erna v. S., jüngster Bruder Wilhelm. 2. R. v. l. Gustel v. S. (mit Rad), Mia v. Boetticher, Oskar (Vater von Reinhold) das Silberpaar Johanna Genevra und Onkel Carl Gustav, Ehepaar Alexandra und Theodor v. Boetticher, Spirgen, Hanna Becker (Reinholds spätere Frau), davor sitzend Reinhold v. S. 3. R. v. l. E. Fehre, Ernst u. Ina, Konrad v. B., Sasching v. B., ältester Bruder Oskar Wilhelm, Ella Elisabeth v. Lieven, Heinrich v. S., Lucie v. S. 4. R. v. 1. Hugo v. B., Lilly v. S., Herrmann, oberste R. Sascha geb. v. S., Edith Schmidt, Alma v. S. 


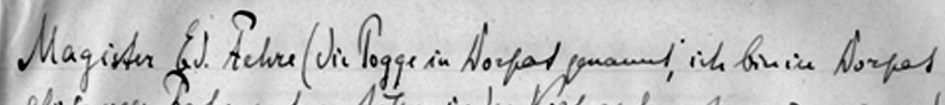

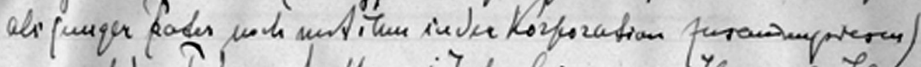

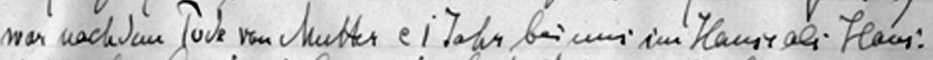

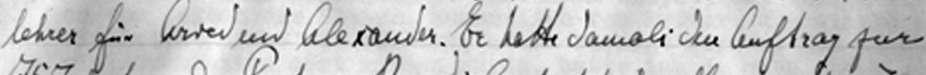

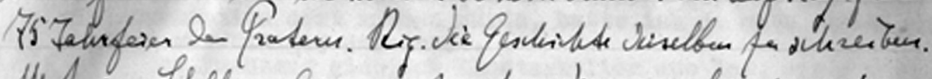

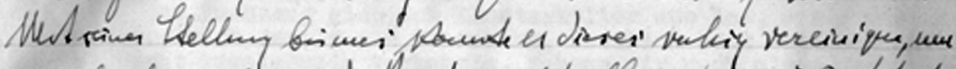

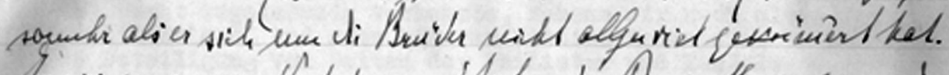

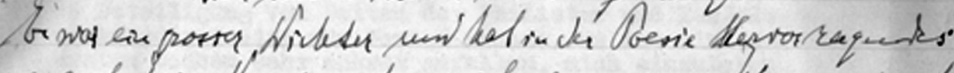

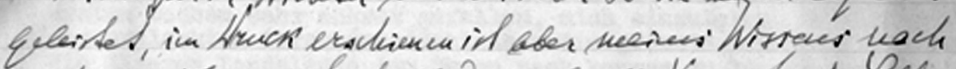

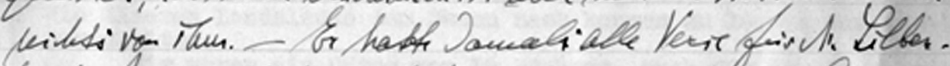

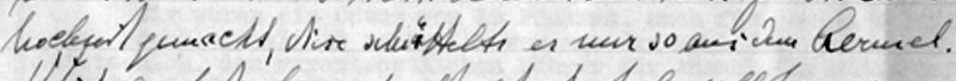

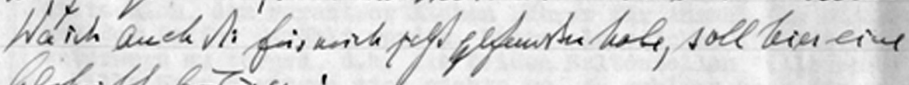
borkifs briven:

Hearer:

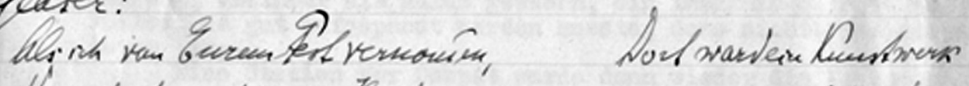

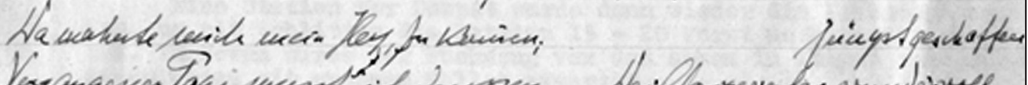

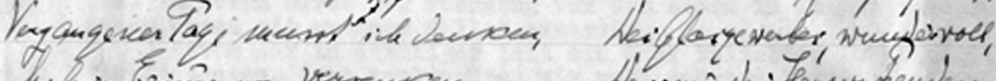
terik in Erivenuzg versancen,

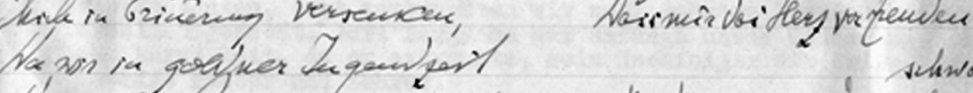

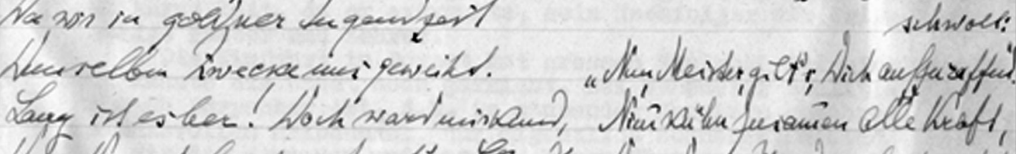

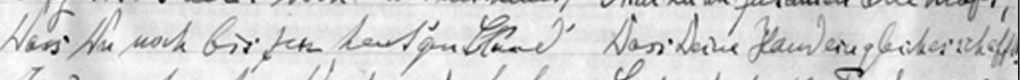

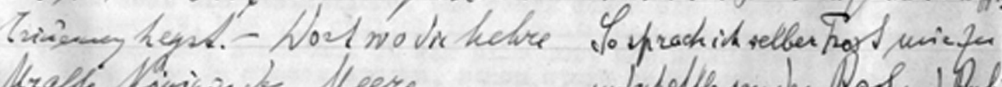

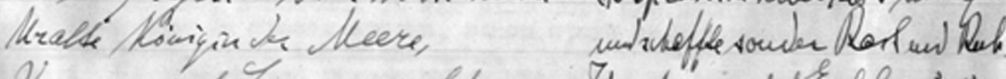

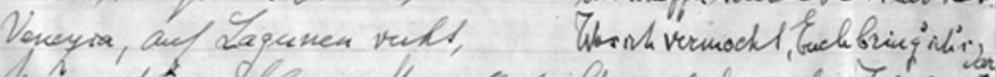

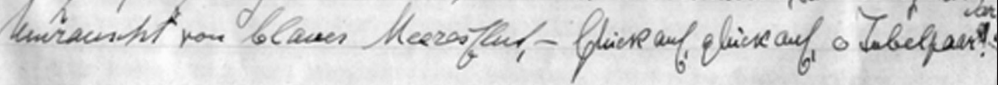

Abb. 4. Gedicht von Magister Fehre zur Übergabe von einer rubinrot und golden gehaltenen Glas-Obstschale von R. v. S. 


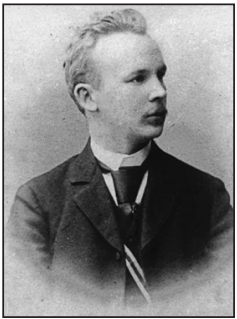

Abb. 5. Reinhold Alexander, als Student 1894

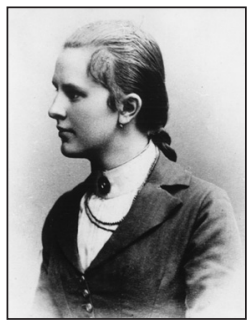

Abb. 6. Hanna, als ich sie kennen lernte

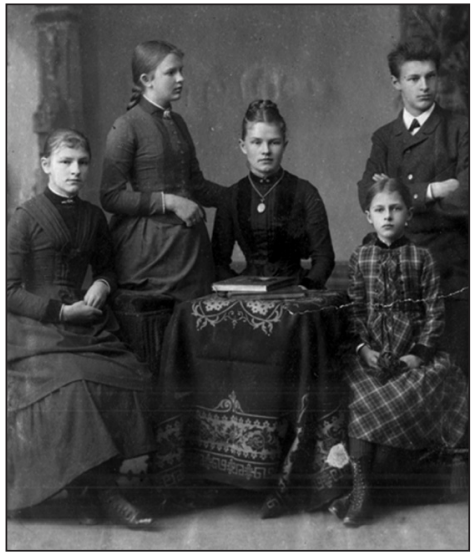

Abb. 7. Die Geschwister Becker, von links: Marie, Hanna, Mathilde, Elsbeth, Rudolf

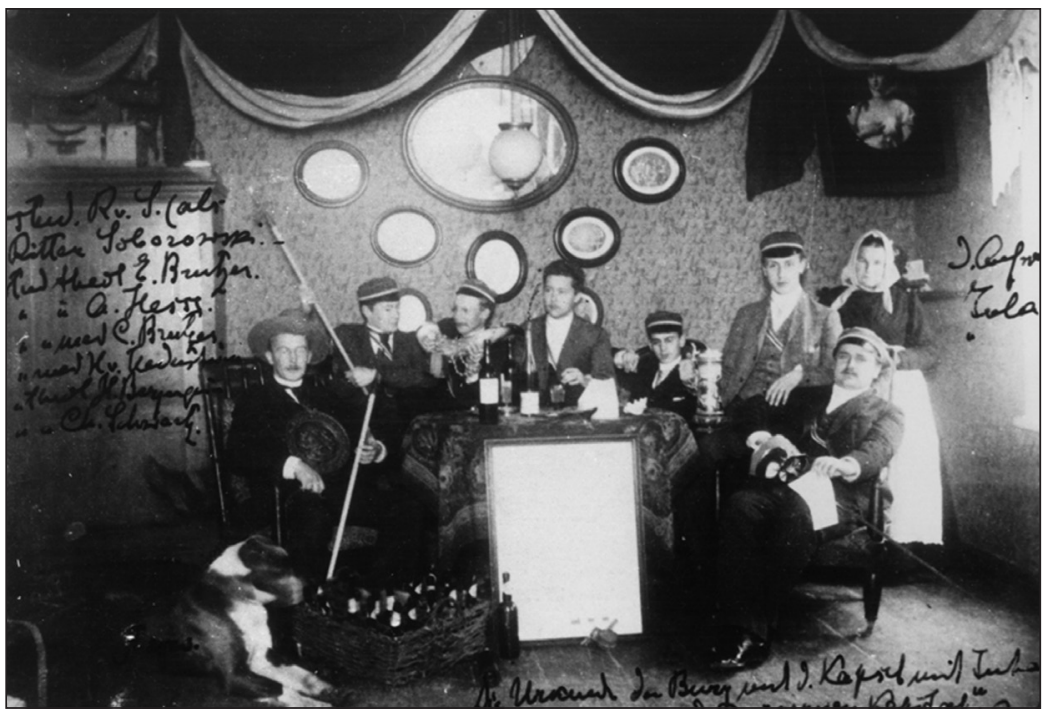

Abb. 8. 30-Jahrfeier der Burg Soborowskerei, Reinhold mit seinen Kommilitonen, v. 1. stud. med. Reinhold v. S. als Ritter Soborowski, stud. theol. E. Brutzer, stud. theol. A. Hesse, stud. med. C. Brutzer, stud. med. H. v. Hedenstöm, stud. theol. H. Bergengrün, stud. theol. Ch. Schwartz, dahinter die Betreuung Jula, l. v. Reinholds Dogge Negro, in der Mitte Urkunde der Burg mit Kapsel Inhalt "Jammerverein Kapital" in Rubel 
Röntgenpionier Dr. med. R. A. von Sengbusch (1869-1944)
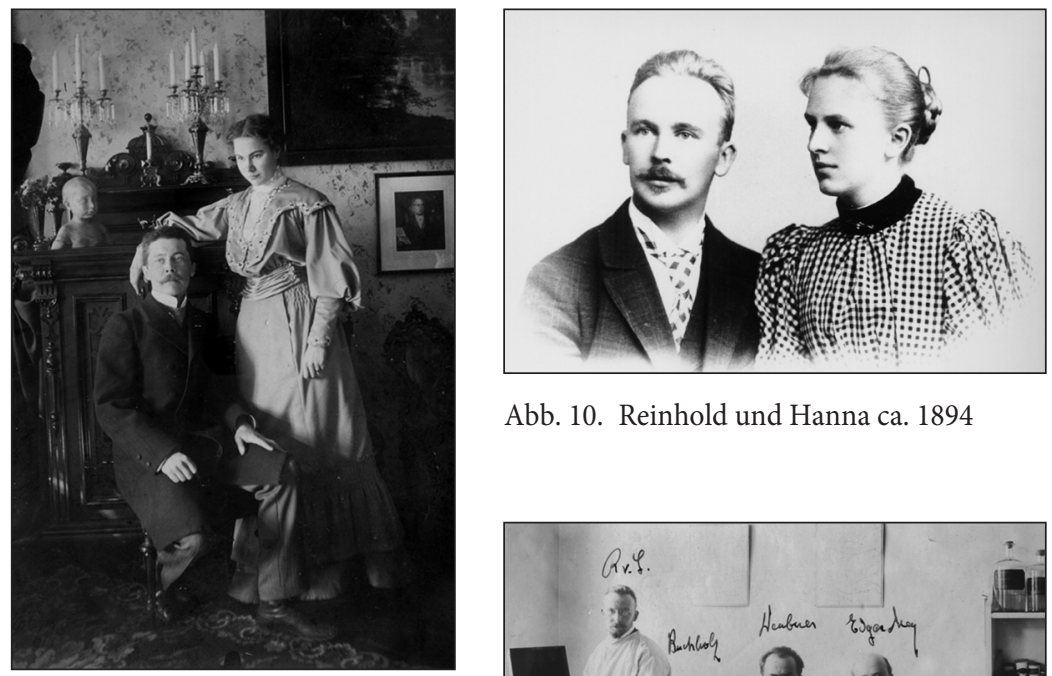

Abb. 10. Reinhold und Hanna ca. 1894

Abb. 9. Dr. med. Karl Brutzer und seine Frau Irma, geb. Westermann
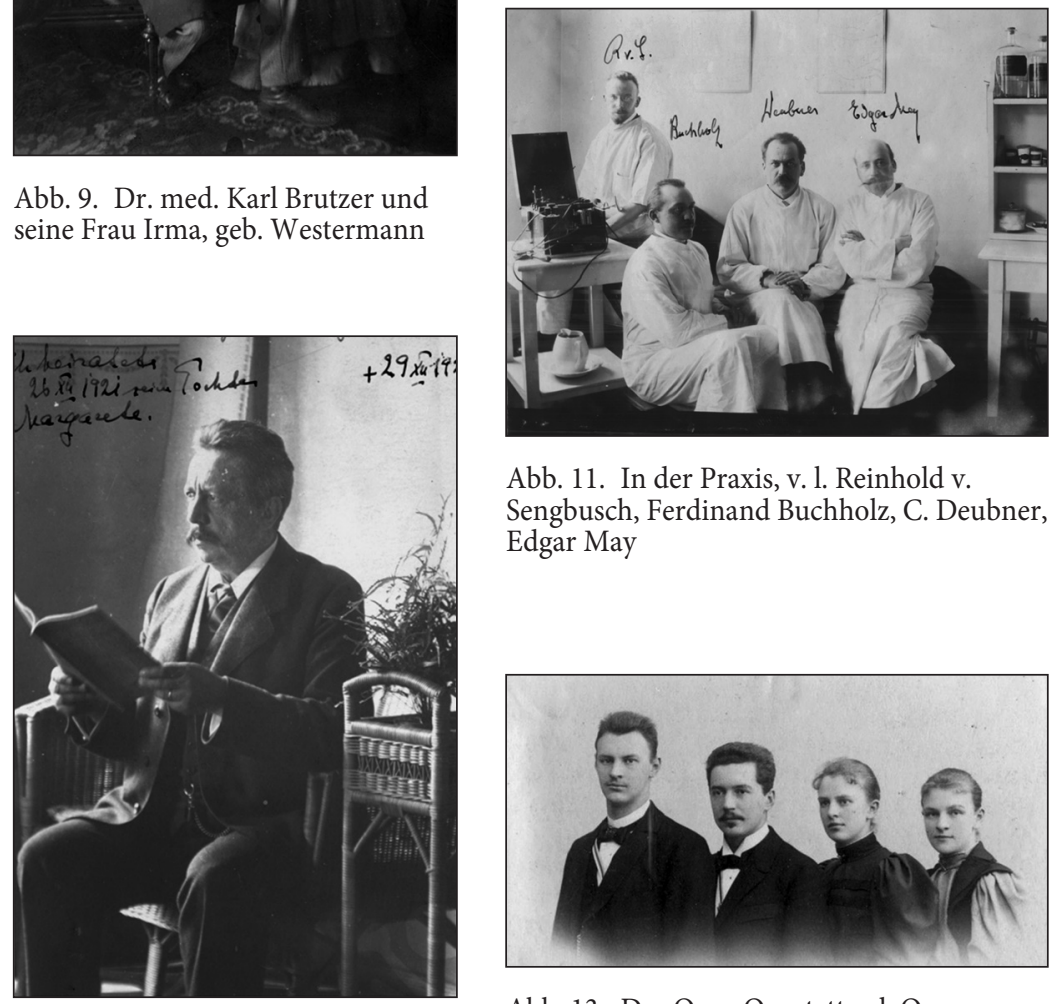

Abb. 11. In der Praxis, v. l. Reinhold v.

Sengbusch, Ferdinand Buchholz, C. Deubner, Edgar May

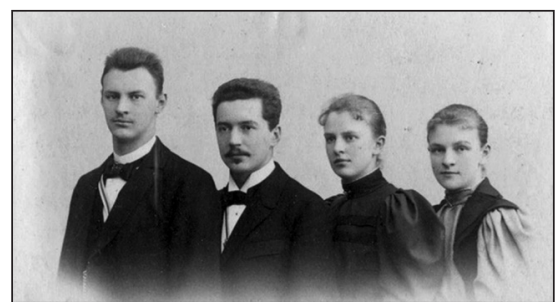

Abb. 13. Das Oger-Quartett v. 1. Oscar Erdmann, Bruder Rudolf, Hanna, Schwester Marie 


\section{W. von Sengbusch}

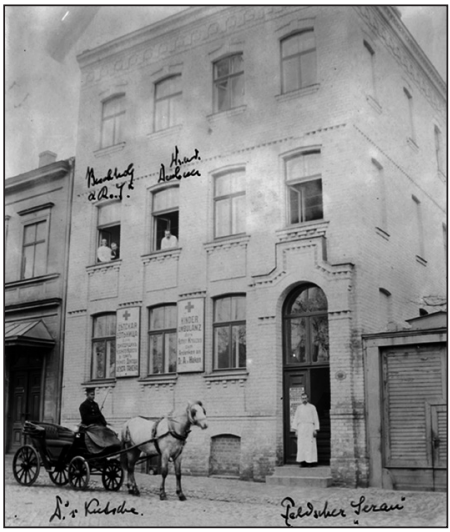

Abb. 14. Kinderambulanz des Roten Kreuzes in der Schulenstraße zum Andenken an Dr. August Haken, im l. Fenster links F. Buchholz, rechts R. v. Sengbusch, im r. Fenster Dr. med. Carl Deubner, unten Feldscher "Serau" und der wohl gemeinsame Kutscher der Ärzte

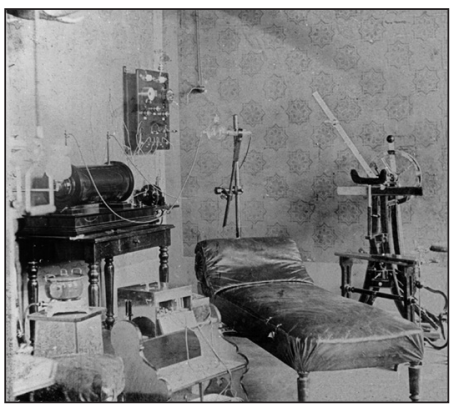

Abb. 17. Praxis von Reinhold v. S., hinter der UntersuchungsChaiselongue erster Röntgenapparat von ca. 1900, vermutlich mit kleiner Müller-Röntgen-Röhre, links dazugehöriger Induktionsapparat, rechts orthopädischer Pendelapparat

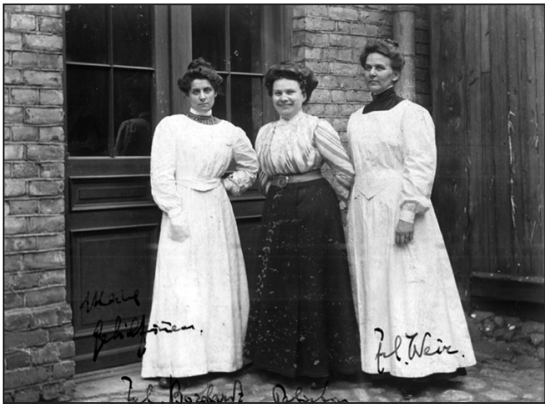

Abb. 15. Mitarbeiterinnen, die beim Einstellen der Apparate und bei der Damenstunde halfen, v. 1. Fräulein Borchert, eine Patientin, Fräulein Weir

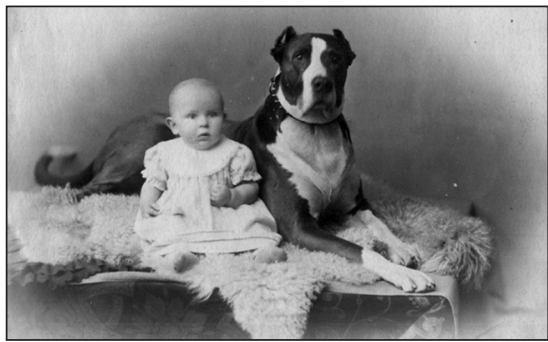

Abb. 16. Gut beschützt - Reini mit Negro, Weihnachten 1898

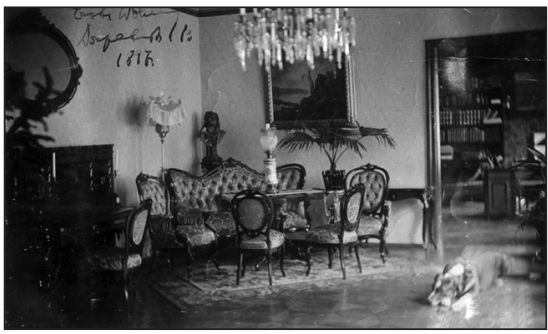

Abb. 18. 1897 - erstes Wohnzimmer in der Dorpaterstraße 13 


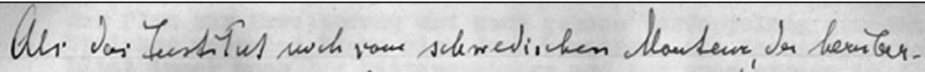

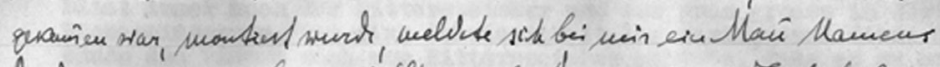

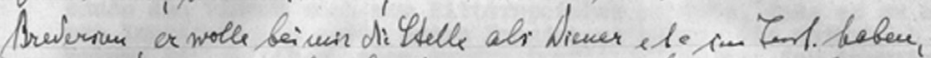

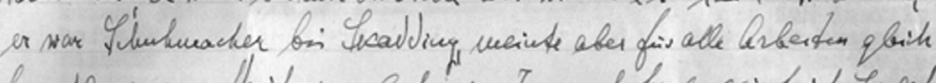

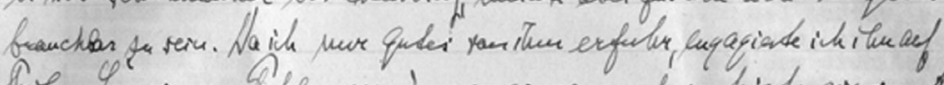

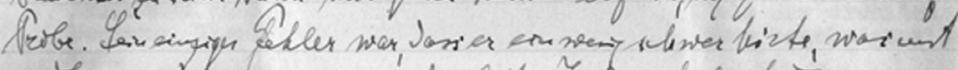

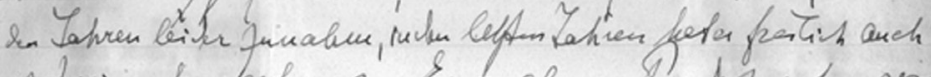

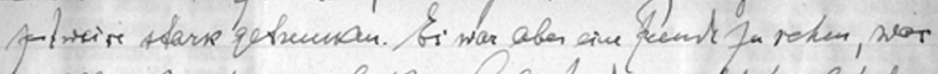

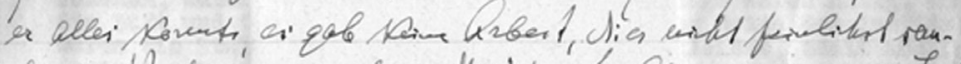

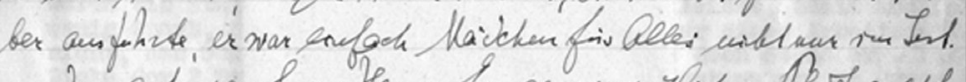

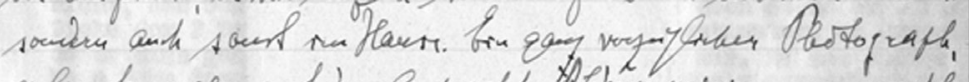

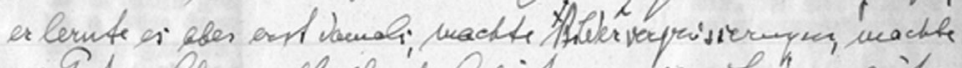

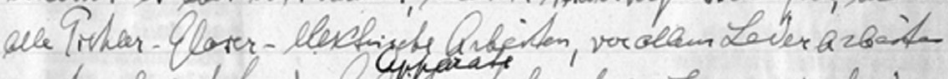

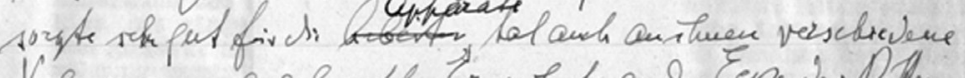

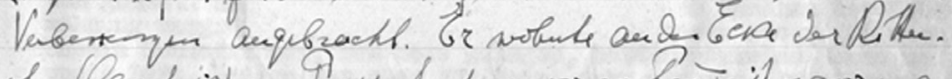

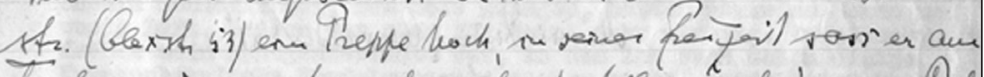

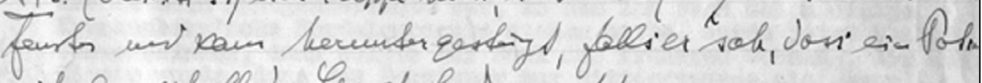

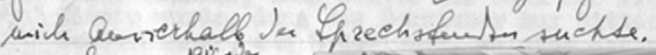

alichinch starle $19 i 7$

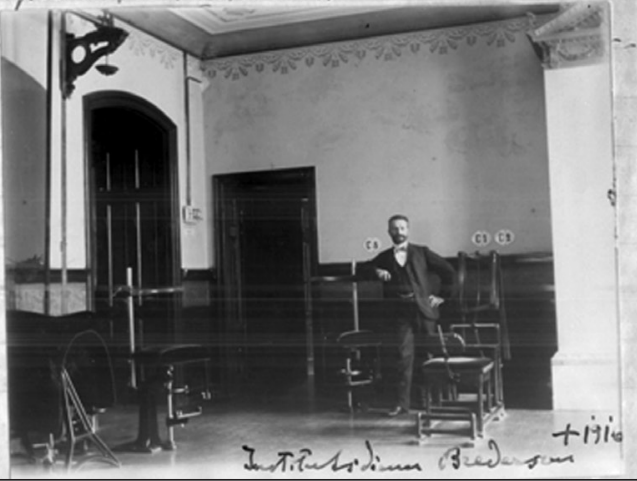

Abb. 19. Originalseite der handschriftlichen Erinnerungen von Reinhold v. S., auf dem Bild sein treuer Institutsdiener und technisches Universal-Genie Brederson, 1910 


\section{W. von Sengbusch}
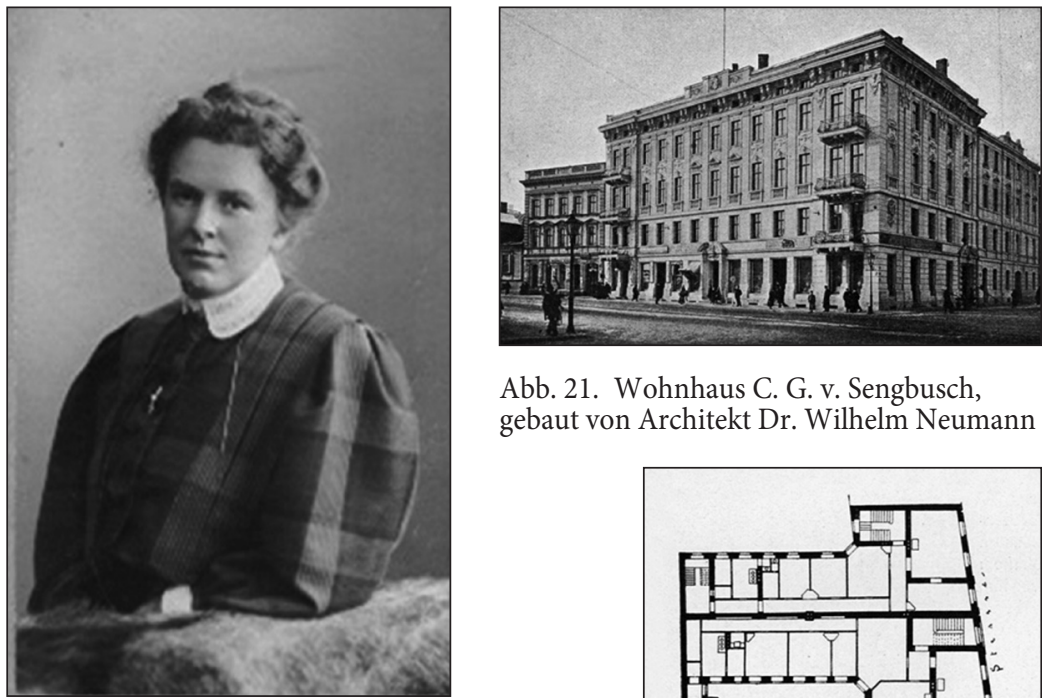

Abb. 21. Wohnhaus C. G. v. Sengbusch, gebaut von Architekt Dr. Wilhelm Neumann

Abb. 20. Hanna um 1907

Abb. 22. Grundriss 1. OG, unten Praxis Reinhold v. S.
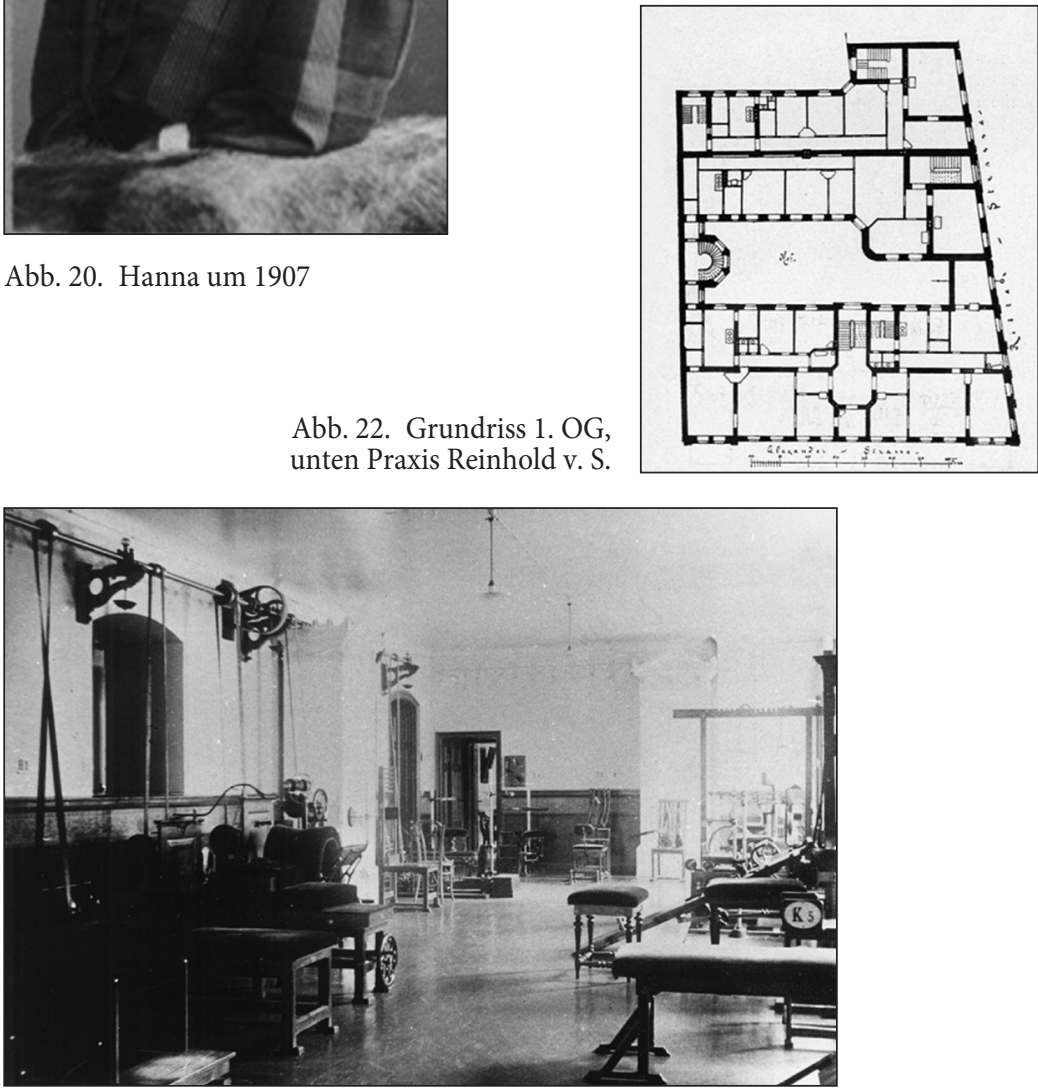

Abb. 23. Zander Institut Rīga - Saal II von der Tür des Röntgenraumes 
Röntgenpionier Dr. med. R. A. von Sengbusch (1869-1944)

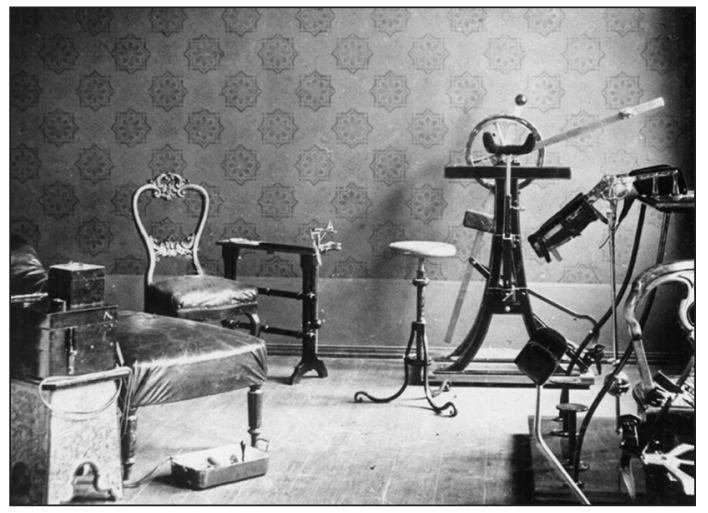

Abb. 24, orthopädische

Pendelapparate um 1900

Abb. 25. Orthopädisches Zander Institut Riga von Dr. med. Reinhold v. S. um 1900 Saal I - links aktive, rechts passive Apparate
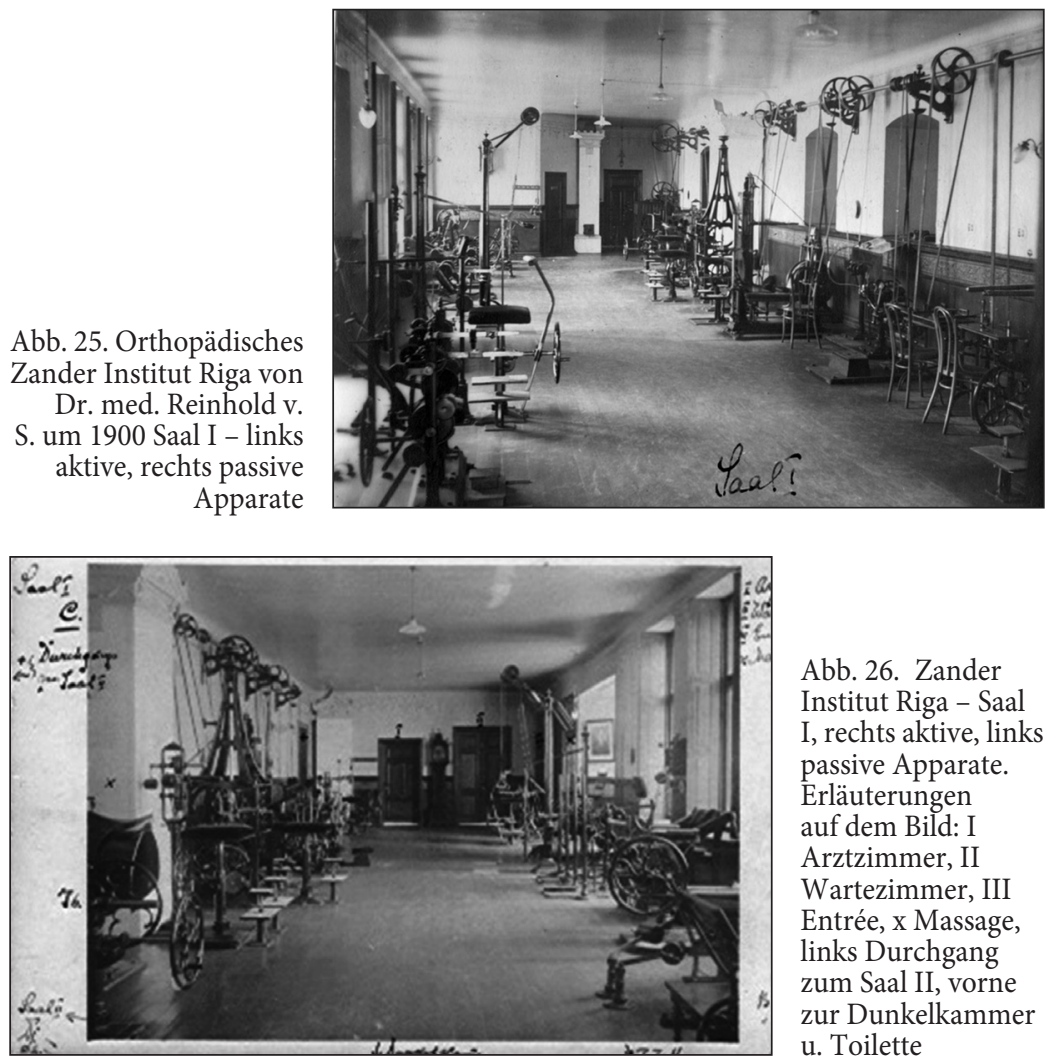

Abb. 26. Zander Institut Riga - Saal I, rechts aktive, links passive Apparate. Erläuterungen auf dem Bild: I Arztzimmer, II Wartezimmer, III Entrée, x Massage, links Durchgang zum Saal II, vorne zur Dunkelkammer u. Toilette 


\section{W. von Sengbusch}

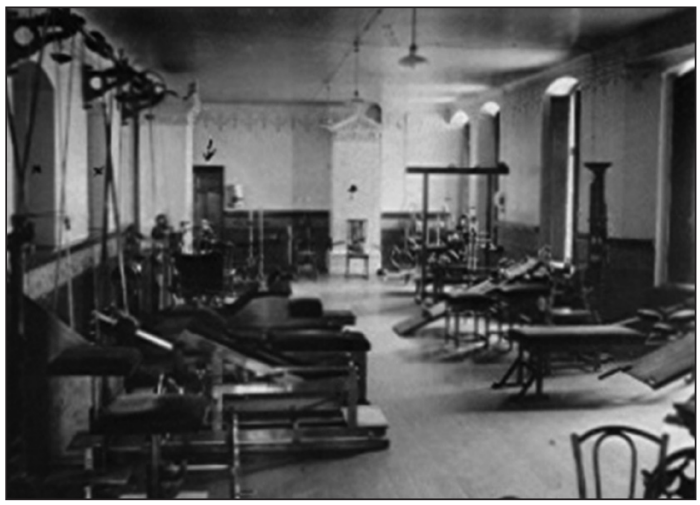

Abb. 27. Zander Institut Rìga - Saal II, hinten Ofen für den Saal

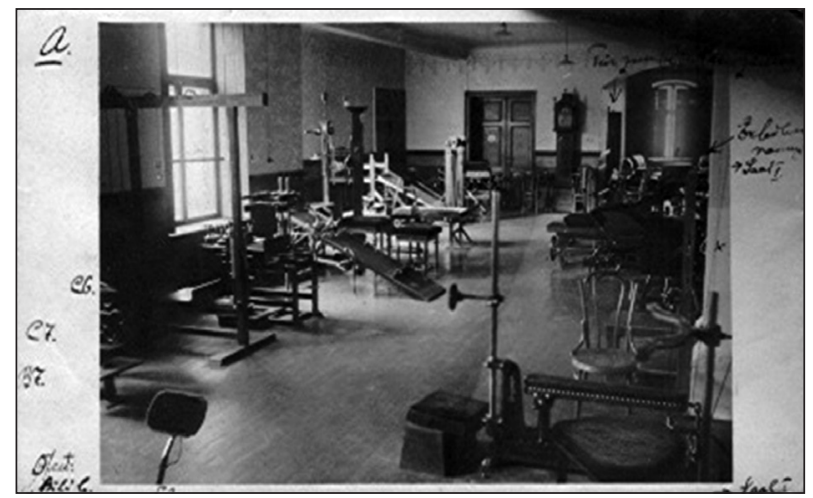

Abb. 28. Zander Institut Rīga Saal II, Gegenseite zu Bild 26. Erläuterungen auf dem Bild, hinten rechts Tür zum Röntgenzimmer, rechts zum Saal I

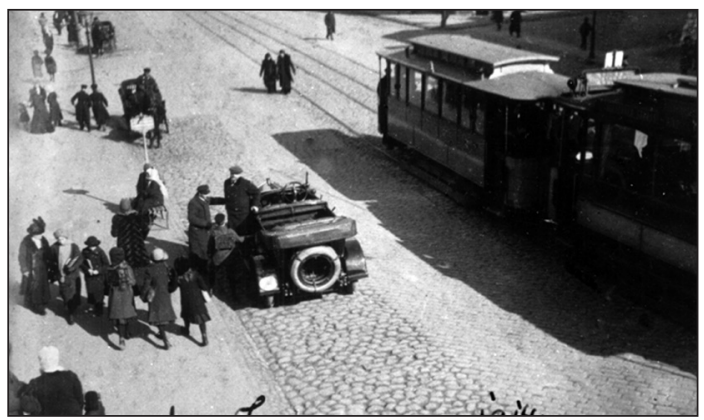

Abb. 29. 1914 - Reinhold aus seinem Hansa

Wagen aussteigend, links Chauffeur Ludwig, davor Hans Buchholz 
Röntgenpionier Dr. med. R. A. von Sengbusch (1869-1944)

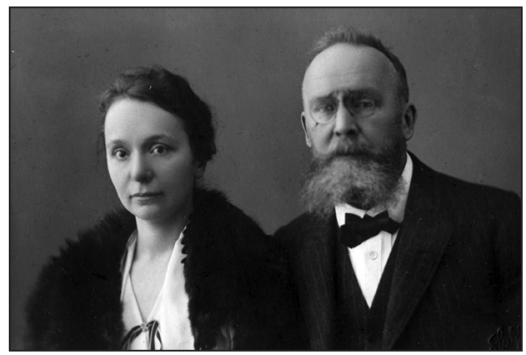

Abb. 30. Reinhold v. Sengbusch mit seiner zweiten Frau Grete, geb. Schütz

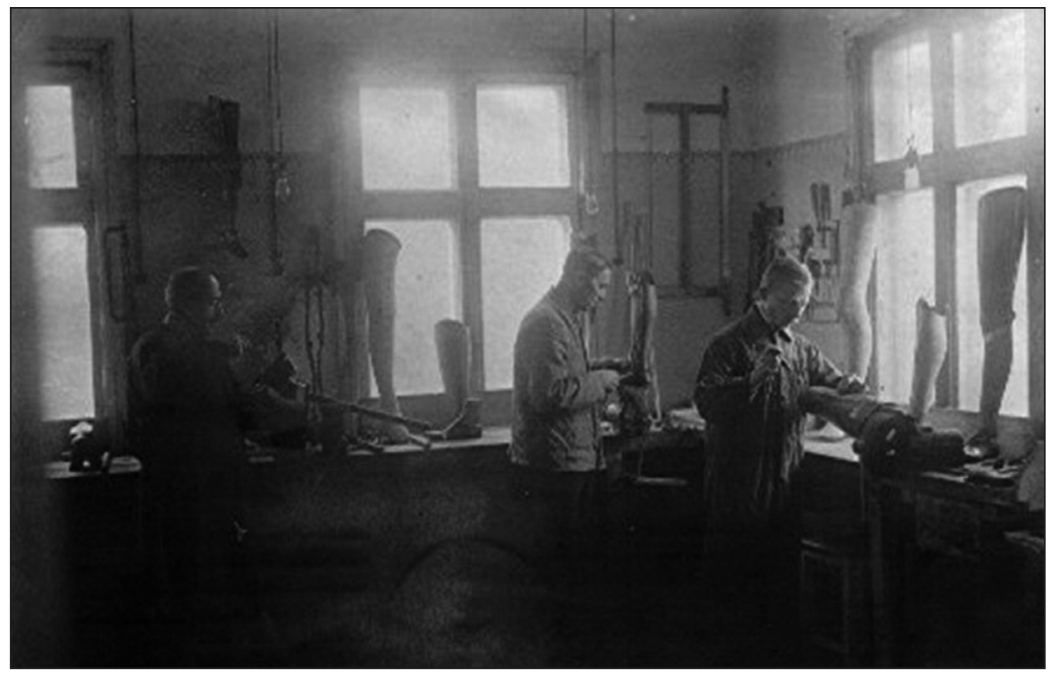

Abb. 31. Prothesenwerkstatt 1923-1932, in der Mitte sein Chauffeur Ludwig

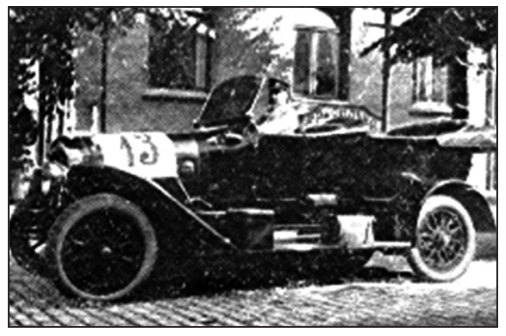

Abb. 32. Vor der Abfahrt zur Fernfahrt Riga-Moskau 1914. Dr. R. von Sengbusch auf seinem 12/36 HP Hansa Wagen 


\section{W. von Sengbusch}

mich an seinen Pfosten der Schwachstromleitung die nötige Starkstromleitung anzubringen, dieselbe dann auf eigens für mich gestellte drei Pfosten bis an das Haus Ecke Alexander- und Ritterstraße [Bruninieku iela] heranzuleiten.

Es war natürlich dabei alles streng verboten, sowohl das Anbringen von Starkstromleitungsdrähten an Pfosten, die nur zur Benutzung für Schwachstrom vorhanden sind, als auch das Queren meiner Leitung über die Ritterstr. Die Sache wurde aber so ausgeführt; ich konnte nun definitiv Auftrag nach Berlin geben für den Induktorapparat, betrieben mit Gleichstrom 120 Volt.

Im Oktober 1899 war ich dann zu Hause Alexanderstraße 51, eine Treppe hoch, eingerichtet. An Stelle von Pohrt übernahm ich den Krankenhausbetrieb erst im Juli 1901, wo ich die Arbeit in den ersten Monaten noch in der Ambulanz des Krankenhauses fortsetzen musste, dort war aber die Arbeit furchtbar unbequem. Die Dunkelkammereinrichtung war auch entsetzlich provisorisch. Ich drang darauf im Interesse der Arbeit, das Kabinett praktischer und bequemer für mich und die Patienten unterzubringen, so wurden die Parterreräume, die in Baracke 7 vorhanden waren, für mich gut ausgebaut.

$\mathrm{Zu}$ Hause musste ich mir die Dunkelkammer erst in der Badestube einrichten, was auch als Notbehelf gelten musste. Als mein Zander-Institut unten eingerichtet wurde, war das Röntgenzimmer das letzte kleine Zimmer, welches in den oberen Etagen Badezimmer war, später ist dieses meine sehr bequem eingerichtete Dunkelkammer geworden, während zum Röntgenkabinett das große Zimmer vorne wurde, das jetzt meine technische Werkstatt ist. Bei Einrichtung meines Institutes 1901 war zum Glück schon der Büngnerhof fertig.

Dieses große Gebäude hatte im Keller seine eigene elektrische Zentrale. Ein Gesuch an das Betriebsamt der Stadt zwecks einer Erlaubnis, mir von dort die Legung einer Leitung zu gestatten, wurde prompt mit der Motivierung abgewiesen, dass es absolut nicht gestattet werden könne, weil zu gefährlich, über die Straße [Säulenstraße - Stabu iela] eine Starkstromleitung zu ziehen. Also wurde sehr bald auch diese Leitung gegen ausführliches Verbot der Stadtverwaltung gelegt. Am Brieger'schen großen Fabrikschornstein kamen die ersten Isolatoren für die Leitung, ferner wurden im Hammer'schen Grunde (Ecke der Alexander-, Säulen- und Kirchenstraße [Baznìcas iela]) 2 hohe Pfosten, im Mündel'schen Grunde noch einer aufgestellt. Auf diese Weise kam ich zur elektrischen Kraft- und Lichtanlage fürs Institut und das Röntgenkabinett. Da ich von Strytzki nicht soviel Kraft bekommen konnte, wie ich jetzt brauchte, gab ich die [erste] Leitung auf, auch hatte ich damals nur immer bis 6 Uhr den Apparat benutzen können, da seine Akkumulatoren-Anlage zu schwach war, um außer seiner Beleuchtung noch für andere Strom abzugeben.

Eine abermalige Änderung im Betriebe des Institutes musste eintreten nach Vollendung des Städtischen Elektrizitätswerkes. Der Büngnerhof gab seine Zentrale auf, er schloss sich an die Stadt an. Für mich kamen zwei Möglichkeiten in Frage: Bau einer eigenen Zentrale, wie der Büngnerhof sie gehabt hatte, oder 
auch Anschluss an die Stadt. Letzteres hatte den Nachteil, dass ich sowohl meinen Gleichstrommotor, der mir die Zander-Apparate betrieb, gegen Drehstrommotor austauschen musste, ferner die Anschlussapparate zum Elektrisieren und für Vibration ändern musste. Vor allem musste ich mir einen neuen Röntgenapparat für Wechselstromanschluss besorgen, resp., einen großen Transformer anlegen.

Röntgen-Apparate mit Anschluss an Wechselstrom gab es freilich damals schon, sie wurden aber nicht sehr empfohlen. Onkel Gustav, mit dem ich über diese Angelegenheit verhandelte, war nicht abgeneigt zu erstem Plane zu raten; er hatte in seiner Korkenfabrik, die schräg vis-a-vis dem Institute in der Ritterstraße lag, eine Art Gasbeleuchtung, die in Hinsicht der Feuersgefahr nicht ganz einwandfrei war. Bei Ausführung dieses Planes wollte er sowohl für die Fabrik, als auch für seine Privatwohnung von mir elektrische Kraft beziehen. Der Bezug wäre vermittelst eines Kabels unter der Erde gestattet worden. Der Vorteil für mich wäre gewesen, dass ich in meinem Institute nichts hätte zu ändern brauchen. Durch Abgabe meines Stromes an Onkel Gustav wäre ich auch gut auf meine Kosten gekommen. Ein Maschinist hätte angestellt werden müssen, Onkel hätte ihm drüben ein Zimmer gegeben, ich ihn gagieren müsse.

Von der A. E. G. ließ ich mir Kostenanschläge machen, leider aber auch von einem Ingenieur Fetting, der mir sehr empfohlen worden war. Eine wenig gebrauchte derartige Anlage, behauptete er, in Petersburg an der Hand zu haben. Er zeigte mir auch eine hierüber lautende Korrespondenz, nach vielem Hin und Her entschloss ich mich zu seinem Plane. Es wurde der dazu gut brauchbare Kellerraum hergerichtet, mit einer guten Steindiele versehen, auch die Wände abgeputzt. Fetting bekam eine Anzahlung zwecks Kaufes und Überführung der Anlage aus Petersburg, und nach einigen Wochen war ich der Hereingefallene, ich erfuhr aus sicherer Quelle, dass er mit diesem Gelde - 3000 Rubel hatte ich ihm ausgezahlt - eine Lokomobile, die schon lange auf dem Güterbahnhofe unausgelöst gestanden, herausgekauft habe, sie auf Hasenholm, wo er eine kleine Sägemühle gepachtet hatte, aufgestellt habe und für die Regierung, mit der er einen Kontrakt auf Lieferung von Schienenschwellen gehabt, angefangen habe zu arbeiten. Es war die Zeit des Russisch-Japanischen Krieges. Nach einem gegen ihn angestrengten Prozess kam es dann zum Krach, er wurde für insolvent erklärt und ich hatte das Nachsehen. Bei der endgültigen Liquidation seines Geschäftes habe ich nach Jahren 300 Rubel ausgezahlt erhalten.

Nun entschloss ich mich schnell, ließ mir von der A. E. G. den Stadtanschluss herstellen. Im Keller genau unter dem Röntgenkabinett, das sich damals schon im zweiten Teile des Institutes befand, wurde ein Umformeraggregat aufgestellt. Jedes Mal vor einer Röntgenuntersuchung musste ich es einschalten und auf richtige Tourenzahl bringen, was ich genau an Messapparaten im Zimmer kontrollieren konnte. Der 3-stiftige Quecksilberunterbrecher stand in der kleinen Kammer unter der Treppe, von dort führten die vier Leitungen zur Schalttafel 


\section{W. von Sengbusch}

im Röntgenzimmer. Einen kleinen Transformator für den Elektrisierapparat ließ ich aus Berlin kommen. Der große Gleichstrommotor wurde durch einen etwas stärkeren Wechselstrommotor ersetzt; so konnte ich nach einigen Wochen den Betrieb wieder voll aufnehmen. Heutigentages ist dieses wohl alles viel leichter und bequemer.

Im Krankenhause bekam ich ca. zur gleichen Zeit, vielleicht etwas früher, einen ganz neuen Apparat, mit schönen, praktischen Nebenapparaten, der AlbersSchönberg'schen Compressionsblende, die ich zu Hause auch schon hatte und die sich, z. B., bei Nierenaufnahmen vorzüglich bewährte. Auch den großen Röhrenkasten und den Untersuchungsstuhl, die notwendig waren für die Lungen- und Herzuntersuchungen, aber dann noch das Trochoskop zur Untersuchung von Patienten im Liegen. Dieses hatte ich nicht, bei mir war kein Platz vorhanden.

\section{Ein paar medizinische Beispiele}

Ich erinnere mich aus dieser Zeit eines sehr hübschen Falles, ich habe keinen derartigen in der Literatur gefunden. Ein 13-jähriger Knabe hatte sich mit einem Monte-Christo eine Verletzung der Brust zugezogen, er wurde von Bergmann ins Röntgenkabinett geschickt. Bei der gewöhnlichen Untersuchung im Sitzen, auch bei einer Plattenaufnahme konnte ich die kleine Kugel nicht nachweisen, erst die Untersuchung auf dem Trochoskop brachte Aufklärung. Ich sah nun deutlich die Kugel in der linken Herzkammer, es war interessant das Vibrieren, das Auf- und Abtanzen des kleinen Projektils bei jedem Zusammenziehen und Erschlaffen des Herzmuskels zu beobachten. Genau dem Pulse entsprechend tanzte sie hin und her. Ich glaube, es war das erste Mal, dass Bergmann damals herunterkam. Er war etwas skeptisch, als ich ihm über den Befund referierte, überzeugte sich aber selbst von der Richtigkeit. Er hat den Knaben dann mit gutem Erfolge operiert. Die Kugel wurde entfernt. Nach Eröffnung der Brusthöhle durch Seitwärtsklappen der Rippen wurde das Herz zur Hälfte vorgezogen, Bergmann meinte jetzt die Einschussöffnung sehen zu können, nun wurden zwei Nähte durch den Herzmuskel geführt, die nach Ausführung des Schnittes die Wunde wieder schließen sollten. Das Herz wurde durch den Assistenten durch Fingerdruck gehalten, jede zu starke Blutung konnte durch Anziehen der Fäden und durch Druck der Finger beeinflusst werden. Nach diesen Vorbereitungen durchtrennte Bergmann den Herzmuskel ganz und ging schnell mit einer Kornzange ein in die Herzkammer, ein mächtiger Blutstrahl schoss empor, wurde aber sofort prompt durch obige Manöver gestoppt. Das Eingehen mit Kornzange aber war nicht nötig gewesen, der Blutstrom selbst warf die Kugel hinaus. Wir hörten zum Glück das Aufschlagen eines Gegenstandes auf den Boden, ich hob die Kugel von der Diele auf und zeigte sie Bergmann, die Wunde wurde sofort geschlossen, der Verlauf war ohne Komplikationen. Da die Kugel schräg durch den Herzmuskel gegangen war, das Geschoß sehr klein war, ist durch die Kompression des Muskels fast kein Blut in den Herzbeutel getreten, es fand sich kein Erguss in ihm. 
An nicht ganz gewöhnlichen Fremdkörpern habe ich im Institute erlebt: Patient aus der Nervenheilanstalt Atgasen hatte einen recht großen Haustürschlüssel verschluckt, gut $12 \mathrm{~cm}$ lang, Griff und Bart ca. $3^{1 / 2} \mathrm{~cm}$ breit, man sah ihn auf den ersten Blick hinter dem Leuchtschirme in der Speiseröhre steckend, der Griff, drei Fingerbreit vom Mageneingang entfernt.

Patientin aus der Anstalt Thabor bei Mitau: verschluckter, großer, zinnerner Speiselöffel, der Löffel war erst künstlich von Patientin etwas zusammengedrückt worden, der Stiel und vorne der Löffel gut $3 \mathrm{~cm}$ breit, ca. $3 / 4$ der unteren Partie der Speiseröhre einnehmend. Die Patientin wurde von Bergmann operiert, es wurde seitlich der Schnitt in die Speiseröhre gemacht, der Löffel durch lange Kornzangen entfernt.

Diese Patientin wurde nach ca. zwei Wochen, die Wunde war schon vollkommen verheilt, abermals, jetzt im Krankenhause, von mir geröntgt, es fehlten auf ihrem Bettische zwei Kaffeelöffel - beide fanden sich bei ihr, der eine schon im Magen, der andere noch in der Speiseröhre. Es fand sich aber noch als Nebenbefund (ich hatte dieses Mal eine Platte gemacht) eine ca. $6 \mathrm{~cm}$ lange, dicke Stopfnadel, die aber nicht mehr im Magen zu sitzen schien. Bei der Operation fand sich diese Nadel dann nach einigem Suchen und Abtasten der Gegend im großen Netze vor, sie war also aus dem Magen und Darm ausgewandert. Glatte Heilung nach Entfernung aller dieser Gegenstände.

Als nächsten Fall folgender: Mann aus Atgasen, er hatte am Abend vorher einer ihn besuchenden Dame eine goldene Uhr nebst kurzer goldener Kette von der Bluse abgerissen, die er ohne weiteres verschluckte. Bei der Durchleuchtung fanden sich Uhr und Kette bereits im Darm, sie sind nach einigen Tagen auf natürlichem Wege abgegangen. Leider hatte der College, der mir den Patienten schickte, nicht daran gedacht, bald darauf den Leib mit dem Ohre abzuhorchen, es wäre interessant gewesen festzustellen, ob und wo man das Ticken der Uhr hätte hören können und ob man das Vorwärtsschreiten derselben im Darme nicht hätte verfolgen können. Als ich den Sitz der Uhr genau konstatiert hatte, muss sie wohl schon gestanden haben, denn ich konnte kein Ticken derselben mehr hören.

Diese Fälle gaben alle ein günstiges Resultat falls man nicht abwartete, sondern sie möglichst bald operierte. Durch längeres Abwarten bilden sich Drucknekrosen, es kommt zu Eiterungen, zu Abscessbildung hinter der Speiseröhre und gehen die Kinder zu Grunde; auch sollen keine Versuche mit unblutiger Extraktion gemacht werden.

Da der Betrieb im Krankenhause immer mehr zunahm, die zwei Abende in der Woche zu denen ich mich verpflichtet hatte, schon lange nicht mehr ausreichten, so dass ich öfter auch schon mehr hingegangen war, wurde der Entschluss gefasst, eine Schwester von draußen zu engagieren. Durch Empfehlung von Prof. Ludloff bekamen wir auch eine, die ihre Sache sehr gut verstand. Sie blieb nur ca. Vier Monate in Rìga, es hatte aber unter ihr in der letzten Zeit eine Rote-Kreuz-Schwester Emmy gearbeitet, die dann schon recht gut die Arbeit übernehmen konnte. Der 
Hauptgrund, weshalb eine Kraft auch am Tage da sein musste, war der, dass die Collegen der Inneren Abteilung sich immer sehr dafür interessierten, dass ihre Patienten zu der Zeit durchleuchtet werden sollten, wenn sie zugegen sein konnten, also während ihrer Visitenzeit oder anschließend an dieselbe. Ich hatte dann aber keine Zeit, da ich im Institute voll beschäftigt war. Schwester Emmy hat dann später bis zum Krieg auch bei mir von 3-5 Uhr gearbeitet.

\section{Schutzmaßnahmen - so gut wie keine}

Die ersten, ich glaube 2 $1 \frac{1}{2}$ Jahre, habe ich ohne jeden Schutz bei diesen Untersuchungen gearbeitet, ohne irgendetwas Schädliches an den Händen zu spüren. Es waren freilich schon Fälle von recht schweren Schädigungen von Collegen bekannt, doch wurde immer wieder behauptet, dass die Sache individuell ganz verschieden wirke. Einer sei mehr disponiert als ein Anderer, erst langsam brach sich die Anschauung Bahn, dass ein gewisses Quantum Strahlen noch nichts schade, wenn dieses aber überschritten ist, es unweigerlich zu schweren Erscheinungen kommt; auch die Strahlenhärte spielt hierbei eine Rolle.

Onkel Gustav hatte mir, es sehr gut meinend, ein Paar Ritterhandschuhe aus Stahlblech gearbeitet, die ich auch eine Zeit benutzt habe. Innen hatten sie dickes Leder, außen die kettenschuppenartig, übereinander gearbeiteten Stahlblechstreifen. Geholfen haben sie natürlich nichts, oder sehr wenig. Als ich an den Händen erkrankte, kam es pistolenschussartig schnell, alle Finger mit einem Male. An allen bildeten sich etwa im Laufe von zwei Wochen eitrige Entzündungen an den Nägeln, dieselben waren reichlich schmerzhaft, es stießen sich langsam die Fingernägel ab, und kann ich noch froh sein und auch dankbar, dass dieselben, wenn auch zum Teile verkrüppelt, wieder gewachsen sind. Es hat viele Wochen gedauert, bis ich wieder richtig arbeiten, z. B., wieder massieren konnte.

$* * *$

Mein Großvater war der erste der nachfolgenden Akademiker-Generationen in der Familie von Sengbusch, die auf fünf sehr erfolgreichen Kaufmanns-Generationen folgte.

Er hat seinen Tribut an seine Pionierarbeit gezahlt, er hat alle Stadien des Krebses aus seinem Beruf durchlebt. Seine letzten Jahre verbrachte er nach der Aussiedlung 1939 in Posen. Der Röntgen-Krebs verschlimmerte sich bis zur Unerträglichkeit, das Rückgrat wurde zerstört.

Dr. med. Reinhold von Sengbusch verstarb am 26. November 1944 in Posen.

\section{Literaturhinweise}

Die Aerzte Livlands von den ältesten Zeiten bis zur Gegenwart.

Ein biographisches Lexikon nebst einer historischen Einleitung über das Medizinalwesen Livlands. Dr. med. I. Brennsohn. Mitau, 1904. Seite 371 und 372. 
v. Sengbusch, Reinhold Alexander, geb. 14. Juli 1869 zu Riga als Sohn des Konsuls Oskar v. S., bes. daselbst das Stadt-Gymn. Stud. In Jurjew (Dorpat) 9095, Arzt Mai 1895; war 95 bis 96 Volontär-Assist. Am Obuchow-Hosp. in St. Petersburg, setzte seine Studien in Berlin und Wien fort, ist seit 1897 freipr. Arzt in Riga, seit 1901 auch Besitzer eines mediko-mechan. Institut daselbst. Uxor Johanna Anna Becker. Eigene Mitteil.-Matrikelnummer 14405. - Alb. Fr. Rig. 984

dito. Dr. med. I. Brennsohn. Mitau 1905

Seite 298 unter Nr. 984

Reinhold Alexander von Sengbusch, med. 901-95 (14405), geb. 14. Juli 1869 zu Riga, Sohn des Konsuls Oskar v. S. Rigasches Stadt-Gymn. F. O. 9011, B. H., C. C. V. Arzt Mai 1895. - Juli 1895-96 Volontär-Assistenzarzt am ObuchowHospital in St. Petersburg. Studierte 1896 und 97 in Berlin und Wien. Seit 1897 prakt. Arzt in Riga. Besitzer und Leiter eines Röntgenkabinetts und Zanderinstituts. Zugleich jüngerer Ordinator am Krankenhaus des Roten Kreuzes und leitender Arzt des Röntgenkabinetts am Stadt-Krankenhause. Seit Dezember 1904 Glied des Engeren Kreises der lit. prakt. Bürgerverbindung.

Paula Stradiṇa Medicīnas vēstures muzejs

Latvijas medicīnas Jubilejas dati 1994. Rìga, 1993, Seite 12 unter

14. jūlijā - pirms 125 gadiem dzimis ortopēds un numismāts Reinholds ZENGBUŠS (1869, Rīga-1944, Vācijā). Beidzis Tērbatas univ. (1895). Ārsts Rīgā (1897-1939), medikomehāniskā inst. īpašnieks (1901-39). Pētījumi numismātikā.

L. Brennson I. Die Aerzte Livlands. Mitau, 1905. S. 371-372.

Folgende drei Quellen sind nicht bekannt, evtl. aus den Rigaer Stadtblättern.

Seite 290

Auf der Tagesordnung der Sitzung der Gesellschaft praktischer Aerzte zu Rīga am Mittwoch den 28. August stand: Krankenvorstellung durch Dr. Bosse und Demonstration des Skioptikons durch Dr. R. von Sengbusch.

\section{Seite 357}

Auf der Sitzung der Gesellschaft praktischer Aerzte zu Rīga am Mittwoch den 2. Oktober gab Herr Dr. M. Hirschberg (als Gast) eine Demonstration von LepraMoulagen und Dr. v. Sengbusch sprach über Röntgenbilder von Skelettheilen Lepröser, demonstriert mit dem Skioptikon.

\section{Seite 307}

Auf der Sitzung der Gesellschaft praktischer Aerzte zu Rīga am Mittwoch den 1. September sprach Dr. v. Bergmann über: "Tuberculose peritonei" und Dr. O. Brehm, als Gast, gab eine Demonstration, wie auch Dr. v. Sengbusch. 


\section{W. von Sengbusch}

Ekstremitāšu protezēšanas vēsture Latvijā, Rīga 2004.

Die Geschichte der Extremitätenprothetik in Lettland

Bakalaura darbs - darba autore Dainuvite Braivo (stud. apl. 0073001)

Rīgas Stradiṇa universitāte - Rehabilitācijas fakultāte

Darba vadītāji: asoc. prof. Juris Salaks, asoc. prof. Mārcis Krūmiņš.

In dieser Arbeit wird die Entwicklung des Prothesenbaues in Lettland erarbeitet und dabei die Leistungen von Dr. med. Reinhold von Sengbusch rückwirkend eingehend beschrieben.

\section{Anmerkungen}

1 Daugagrivas in Zasulauks, nahe der Gartenstraße (Dārza iela) 3, dem Sommersitz von Conrad Heinrich, Wilhelm und Oscar v. S.

2 Rigaer Drahtindustrie 1874 gegründet, siehe "Riga und seine Bauten - 1903" Seite 413.

3 Ursprünglich das Brederlo Haus, kam 1862 testamentarisch an Wilhelm v. S. und damit in die Familie v. S. Es war dann bis zu seinem Abriss 1938 das Haus Sengbusch. Mit diesem Haus war die Brederlo'schen Gemäldesammlung verbunden.

4 Wilhelm v. Sengbusch * Riga 12.XII.1802, † Riga 12.X.1880 schwedischer Konsul,

5 Oscar v. Sengbusch * Riga 22.VIII.1838 - † 1.I.1900

6 Carl Gustav v. Sengbusch * Riga 9.III.1813 - † Riga 30.V.1924.

7 Er war Dom Museumsinspektor von 1901 bis 1924.

8 Anna Elisabeth v. Sengbusch, geb. Pychlau * Riga 10.III.1847 - † Riga 29.III.1896, Tochter des Ratsherrn Reinhold Pychlau und seiner Frau Karoline, geb. Kröger.

9 Reinhold Ludwig Pychlau * Riga 17.IV.1816 - † 1882.

10 Johann Theodor Pychlau * 12.VI.1790 - †, die Holzsägemühle war gelegen auf Muckenholm, Oskarstraße 7.

11 Christian Alexander Gottschalk v. Sengbusch * Riga 16.VIII.1879 - † Zeulenroda 18.2.1951.

12 1914-1918. Der Zusammenbruch des russischen und des deutschen Kaiserreiches beendete die deutsche Vormachtstellung in den baltischen Provinzen Estland, Livland und Kurland.

13 Wilhelm Oscar v. Sengbusch * Riga 19.VI.1866 - † Riga 11.VI.1919.

14 Carl Brutzer, Enkel von Dr. med. Karl Ernst Brutzer ${ }^{\star}$ Riga 31.I.1794 - † 21.II.1877 und seiner 2. Frau Helene Maria Lamprecht * Riga 4.VI.1811 - Riga $\dagger$ 20.VI.1844, Schwester von Catharina Juliane Lamprecht, der Großmutter von R. v. S., * St. Petersburg 3.XI.1812 - † Riga 24.IX.1856.

15 Johanna (Hanna) Anna Margarete, geb. Becker ${ }^{\star}$ Riga 27.III.1873 - † 20.IV.1908, Tochter von Bernhard Max Julius Becker * Ratzeburg 17.12.1829 - 
$\dagger$ Riga 16.XII.1913 und Laura Bernhardine Rosalie, geb. Engelke * Magdeburg 13.8.1835 - † Riga 28.1.1913.

16 Dr. Gustav Adolf (Ado) Hugo Helmboldt * Riga 8.XII.1867, † 26.V.1919, heiratet am 30.V.1896 die Schwester von Hanna, Marie Bernhardine Bertha geb. Becker ${ }^{\star}$ Riga 26.V.1871 - † Frankfurt a. M. 6.5.1956. Er studierte ein Semester Theologie in Dorpat, wechselt sein Studium und wird Mediziner. Beendete Mai 1894 sein Studium, wird Assistent am Rigaer Krankenhaus in der Chirurgischen Abteilung von Dr. A. v. Bergmann. Februar 1896 wird er frei praktizierender Arzt auf dem Gut Lemberg, kehrt 1898 nach Riga als praktischer Arzt zurück und arbeitet seit April des Jahres auch an dem KinderKrankenhaus des Roten Kreuzes, wird dort 1904 Ordinator.

17 Dr. Ferdinand Friedrich Buchholz * Riga 22.9.1868, $-\dagger 17.8 .1918$, heiratet etwa 1902 die jüngsten Schwester von Hanna, Elsbeth (Elschen/Baby) Magdalena Karoline Becker ${ }^{\star}$ Riga 30.3.1878 - †22.5.1957. Studierte in Dorpat, seit 1893 bis 1896 Assistent am Rigaer Stadt-Krankenhaus und setzte dann seine Studien in Wien, Berlin und Leipzig fort. Seit 1897 Spezialarzt für Kinderkrankheiten in Riga und Ordinator der therapeutischen Abteilung des Roten Kreuz.

18 James Armitstead'sches Kinderhospital, Thorensberg, Mitauer Chaussee 23

19 Chirurgisches Krankenhaus, Ambulanz des Rigaschen Asyls der barmherzigen Schwestern des Roten Kreuzes, Adlerstraße [Ërgḷ iela] Nr. 3.

20 Herbert, Sohn von Christian Schmidt und Lina Pychlau.

21 Lina, Tochter von Reinhold Ludwig Pychlau, Kaufmann 1. Gilde * Riga 17.IV.1816 - † 1882 und Caroline Johanna Kröger * Riga 4.3.1826 - †

Werner von Sengbusch, Dipl. Ing. Architekt

Wiesbadener Straße 26

61462 Königstein/Deutschland 